\title{
Large Angular Jump Mechanism Observed for Hydrogen Bond Exchange in
}

\section{Aqueous Perchlorate Solution}

\author{
Minbiao Ji ${ }^{1,2}$, Michael Odelius ${ }^{3}$, and K. J. Gaffney ${ }^{1, *}$ \\ ${ }^{1}$ PULSE Institute for Ultrafast Energy Science, SLAC National Accelerator Laboratory, \\ Stanford University, Stanford, California, 94305, USA \\ ${ }^{2}$ Department of Physics, Stanford University, Stanford, California, 94305, USA \\ ${ }^{3}$ Fysikum, AlbaNova University Center, Stockholm University, SE-106 91 Stockholm, \\ Sweden
}

\begin{abstract}
:
The mechanism for hydrogen bond (H-bond) switching in solution has remained subject to debate despite extensive experimental and theoretical studies. We have applied polarization-selective multidimensional vibrational spectroscopy to investigate the $\mathrm{H}$ bond exchange mechanism in aqueous $\mathrm{NaClO}_{4}$ solution. The results show that a water molecule shifts its donated H-bonds between water and perchlorate acceptors by means of large, prompt angular rotation. Using a jump-exchange kinetic model, we extract an average jump angle of $49 \pm 4^{\circ}$, in qualitative agreement with the jump angle observed in molecular dynamics simulations of the same aqueous $\mathrm{NaClO}_{4}$ solution.
\end{abstract}


Hydrogen bonds (H-bonds) provide the intermolecular adhesion that dictates the unique properties of liquid water and aqueous solutions. Though H-bonds constrain the local ordering and orientation of molecules in solution, these local H-bond networks disband and reform on the picosecond time scale. This structural lability critically influences chemical and biological transformations. Our understanding of the dynamics of hydrogen bond dissociation and reformation has been transformed by the union of ultrafast vibrational spectroscopy and molecular dynamics simulations (1-14), but the detailed mechanism for H-bond switching in aqueous solution remains uncertain. Recent simulation studies of water and aqueous ionic solutions have proposed that $\mathrm{H}$-bond exchange involves large angular jumps of 60 to $70^{\circ}(1,5)$. However, the substantial complexities inherent in simulating the structural and dynamical properties of water highlight the importance of validating this proposal experimentally.

Multidimensional vibrational correlation 2DIR spectroscopy provides an excellent opportunity to study this class of ultrafast chemical exchange (15-19). Here we apply a variation of this technique (Fig.1) to directly investigate the orientational jump mechanism for H-bond switching in aqueous ionic solutions. The dissolution of $\mathrm{NaClO}_{4}$ in isotopically mixed water generates two deuterated hydroxyl stretch (OD) frequencies: OD groups donating a H-bond to another water molecule $\left(\mathrm{OD}_{\mathrm{w}}\right)$ absorb at $2534 \mathrm{~cm}^{-1}$, whereas $\mathrm{OD}$ groups donating a $\mathrm{H}-$ bond to a perchlorate anion $\left(\mathrm{OD}_{\mathrm{P}}\right)$ absorb at $2633 \mathrm{~cm}^{-1}$, (Fig. 1D). This spectroscopic distinction between the $\mathrm{OD}_{\mathrm{W}}$ and $\mathrm{OD}_{\mathrm{P}}$ provides the opportunity to track H-bond exchange by monitoring the growth in the cross-peak intensity in the time-dependent 2D spectra $(3,4)$, as shown in Fig. 1. Recent studies by Moilanen et al. (3) and Park et al. (4) have used this attribute of the water hydroxyl 
stretch to measure the $\mathrm{H}$-bond exchange rate in aqueous $\mathrm{NaBF}_{4}$ and $\mathrm{NaClO}_{4}$ solutions. These experiments measured the exchange between water-water and water-anion H-bond configurations, but did not directly address the hydroxyl group re-orientation associated with H-bond exchange.

Two advances implemented in the present study have allowed us to extract the orientational jump angle associated with H-bond exchange. First, we have measured the laser polarization dependence of the 2DIR spectra (20). Polarization-selective vibrational pump-probe measurements have been widely utilized to study the orientational dynamics of water molecules $(6,7)$, but these measurements cannot distinguish which water molecules have exchanged their H-bonding configuration. This can only be achieved by introducing an additional spectral dimension. Our work extends prior polarizationselective 2DIR studies of the relative orientation of coupled vibronic states $(21,22)$; specifically, we address the time-dependent change in orientation of the vibrationally excited hydroxyl groups induced by chemical exchange. The second advance is a modification of the kinetic model (23) used to interpret 2DIR spectra, so as to include vibrational transition dipole moment jump-reorientation induced by chemical exchange. Prior data analysis assumed H-bond exchange did not induce jump-reorientation $(3,4)$.

2DIR spectroscopy monitors equilibrium H-bond switching dynamics on a picosecond time scale (15-19) by labeling molecules through resonant excitation at their hydroxyl (OD) stretch frequencies, and then correlating these initial frequencies $\left(\omega_{\tau}\right)$ with the (potentially shifted) stretch frequencies $\left(\omega_{m}\right)$ associated with these same molecules after an experimentally controlled waiting time $\left(T_{W}\right)$. Thus, 2DIR can determine when a vibrationally labeled $\mathrm{OD}_{\mathrm{W}}$ hydroxyl group converts to an $\mathrm{OD}_{\mathrm{P}}$ 
configuration during $T_{W}$. Note that the cross-peak only reflects H-bond exchange events in which the hydroxyl group switches between the $\mathrm{OD}_{\mathrm{W}}$ and $\mathrm{OD}_{\mathrm{P}}$ configurations. By adding polarization selectivity, we furthermore access the orientational dynamics of water molecules that have switched between $\mathrm{OD}_{\mathrm{W}}$ and $\mathrm{OD}_{\mathrm{P}}$ configurations. In the polarization selective 2DIR measurement, the first two pulses that control the frequency labeling of OD stretches have parallel polarizations that preferentially excite transition dipole moments parallel to the excitation polarization. During the $T_{w}$ waiting time, the excited molecules randomize their orientation in addition to undergoing H-bond exchange between the $\mathrm{OD}_{\mathrm{W}}$ and $\mathrm{OD}_{\mathrm{P}}$ configurations (Fig. 1A). If the H-bond exchange minimally perturbs the vibrationally excited hydroxyl group orientation, both the diagonal and the cross-peak intensities will exhibit similar polarization dependence. However, if the molecules exchange via large angular jumps, the cross-peak signal that results solely from $\mathrm{OD}_{\mathrm{W}}-\mathrm{OD}_{\mathrm{P}}$ exchanged populations will show distinctly different polarization dependence from the diagonal peaks (Fig. 1B, C).

Figure 2 shows the $T_{W}$-dependent polarization selective 2DIR spectra for aqueous $6 \mathrm{M}$ $\mathrm{NaClO}_{4}$. The positive peaks shown in red along the diagonal $\left(\omega_{\tau}=\omega_{m}\right)$ result from the fundamental vibrational transition $(v=0 \rightarrow 1)$ within each $\mathrm{H}$-bond configuration. We label the peak volumes $I_{l l m m}^{(i j)}$ for the diagonal $(i=j)$ and the off diagonal $(i \neq j)$ peaks: $i$ and $j$ refer to the H-bond configurations associated with $\omega_{\tau}$ and $\omega_{m}$ and $l$ and $m$ refer to the laser polarization. The cross-peak at $\left(\omega_{\tau}, \omega_{m}\right)=(2534,2633) \mathrm{cm}^{-1}$ results from $\mathrm{OD}_{\mathrm{W}}$ switching to $\mathrm{OD}_{\mathrm{P}}, I_{l l m m}^{(W P)}$. Since this H-bond exchange occurs at equilibrium, the exchange process from $\mathrm{OD}_{\mathrm{P}}$ to $\mathrm{OD}_{\mathrm{W}}$ will also generate a cross-peak $I_{l l m m}^{(P W)}$ at 
$\left(\omega_{\tau}, \omega_{m}\right)=(2633,2534) \mathrm{cm}^{-1}$ with $I_{l l m m}^{(P W)}=I_{l l m m}^{(W P)}$. Experimentally, the $v=1 \rightarrow 2$ excited state absorption of $\mathrm{OD}_{\mathrm{P}}$ obscures this cross-peak (4), so we use the $I_{l l m m}^{(W P)}$ signal to characterize the jump angle. The vibrationally excited molecules that have switched between the $\mathrm{OD}_{\mathrm{P}}$ and $\mathrm{OD}_{\mathrm{W}}$ configurations project nearly equivalent signal intensities for perpendicular and parallel polarizations. As will be shown, this equivalence reflects the large angular jumps that lead to H-bond switching.

Figure 3 further highlights the polarization-dependent population dynamics by comparing the peak volumes for the isotropic $\left(I_{i s o}^{(i j)}=I_{z z z z}^{(i j)}+2 I_{z z y y}^{(i j)}\right)$ and anisotropic $\left(I_{\text {aniso }}^{(i j)}=I_{z z z z}^{(i j)}-I_{z z y y}^{(i j)}\right)$ signals at a diagonal-peak $(i j=P P)$ and a cross-peak $(i j=W P)$. The $I_{i s o}^{(P P)}$ signal reflects the population decay of the $\mathrm{OD}_{\mathrm{P}}$ configuration, whereas $I_{\text {aniso }}^{(P P)}$ reflects population relaxation and orientational randomization of the $\mathrm{OD}_{\mathrm{P}}$ configuration. The $I_{i s o}^{(W P)}$ signal rise time reflects the rate of $\mathrm{H}$-bond switching, whereas the decay results primarily from $\mathrm{OD}_{\mathrm{P}}$ population relaxation. Unlike prior measurements of the H-bond switching time $(3,4), I_{i s o}^{(i j)}$ decouples the orientational dynamics from the H-bond configurational switching and so provides a superior measure of the exchange rate. The $I_{\text {aniso }}^{(W P)}$ signal clearly shows the very small dependence of the cross-peak intensity on the laser polarization. The method used to extract the peak volumes can be found elsewhere $(3,23)$.

We modeled the polarization-selective 2DIR spectra using diagrammatic perturbation theory and an angular jump-exchange kinetic model. This provides a framework for interpreting the time $\left(T_{W}\right)$ and angle $(\Omega)$ dependent excited state population $N_{i}\left(T_{W}\right)$, orientation, and spectral diffusion dynamics $(20,23,24)$. The 
simplest variant of the angular jump-exchange kinetic model presumes that an orientational jump through angle $\Theta$ accompanies $\mathrm{H}$-bond exchange between the $\mathrm{OD}_{\mathrm{W}}$ and $\mathrm{OD}_{\mathrm{P}}$ configurations, while reorientation of hydroxyl groups occurs diffusively within a particular H-bond configuration. The model requires the angular rate of change during an angle jump to be much larger than the angular rate of change induced by orientational diffusion. As will be discussed, this requirement is consistent with our experimental and simulation results.

The angular jump-exchange kinetic model attributes the dynamics to (i) population decay with decay rates $k_{W}$ and $k_{P}$, (ii) orientational diffusion with diffusion constants $D_{W}$ and $D_{P}$, and (iii) chemical exchange with rate constants $k_{W-P}$ and $k_{P-W}$ and a H-bond exchange jump angle $\Theta$. This model leads to ensemble averaged populations as a function of $T_{W}$ and laser polarization. For the $S_{z z z z}$ and $S_{z z y y}$ geometries, the polarizationdependent populations can be expressed as in eqs. 1 and 2:

$$
\begin{aligned}
& \left(\begin{array}{c}
N_{W}\left(T_{w}\right) \\
N_{P}\left(T_{w}\right)
\end{array}\right)_{z z z z}=\left(e^{-A \cdot T_{w}}+\frac{4}{5} e^{-B \cdot T_{w}}\right) \times \frac{1}{3}\left(\begin{array}{c}
N_{W}(0) \\
N_{P}(0)
\end{array}\right) \\
& \left(\begin{array}{c}
N_{W}\left(T_{w}\right) \\
N_{P}\left(T_{w}\right)
\end{array}\right)_{z z y y}=\left(e^{-A \cdot T_{w}}-\frac{2}{5} e^{-B \cdot T_{w}}\right) \times \frac{1}{3}\left(\begin{array}{c}
N_{W}(0) \\
N_{P}(0)
\end{array}\right)
\end{aligned}
$$

$A$ and $B$ correspond to matrices that contain all the kinetic coefficients, as well as $\Theta$,

$$
A=\left(\begin{array}{cc}
k_{W}+k_{W-P} & -k_{P-W} \\
-k_{W-P} & k_{P}+k_{P-W}
\end{array}\right), \quad B=\left(\begin{array}{cc}
k_{W}+k_{W-P}+6 D_{W} & -\left\langle P_{2}(\cos \Theta)\right\rangle k_{P-W} \\
-\left\langle P_{2}(\cos \Theta)\right\rangle k_{W-P} & k_{P}+k_{P-W}+6 D_{P}
\end{array}\right)
$$

where $P_{2}(\cos \Theta)=\left(3 \cos ^{2} \Theta-1\right) / 2$ and $\langle\ldots\rangle$ corresponds to an ensemble average. These equations reduce to the exchange kinetic model of Kwak et al. (23) when $\Theta=0^{\circ}$ and can 
be straight forwardly related to the theory of coupled vibrations developed by Golonzka and Tokmakoff (21). The $A$ matrix has no orientational dependence and contributes solely to $I_{i s o}^{(i j)}$ with dynamics determined by the excited state lifetime and the chemical exchange rate. The $B$ matrix contributes solely to $I_{\text {aniso }}^{(i j)}$ with dynamics determined by $\Theta, D_{W}$, and $D_{P}$. As shown in the supporting text, a large jump angle leads to a small $\left\langle P_{2}(\cos \Theta)\right\rangle$ value and the very small cross-peak anisotropy measured experimentally.

We have chosen to present the simplest variant of the jump exchange kinetic model for conceptual clarity, but we have also investigated more sophisticated models. In the supporting text we discuss three critical aspects of the model and demonstrate that the extracted $\Theta$ value is fundamentally insensitive to the distribution of jump angles, the time evolution of the jump angle, and the detailed dynamics of the orientational randomization for hydroxyl groups that remain in a given H-bond configuration.

We employ numerical response function calculations based on the above model to analyze the experimental data. The calculated anisotropic spectra appear in Fig. 2(D). We use standard methods to obtain starting values for the vibrational relaxation, orientational diffusion, and frequency-frequency correlation function parameters $(4,23)$. The fitting of the polarization selective 2DIR spectra allow the extraction of the H-bond exchange rate $k_{W-P}$ and jump angle $\Theta$. The analysis uses global fitting of the polarization-selective 2DIR spectra for $T_{W}$ times of $0.2,1,2,3,4,5$, and 7 ps. Fitting results give $\Theta=49 \pm 4^{\circ}$, $\left(6 D_{W}\right)^{-1}=5 \pm 0.5 \mathrm{ps},\left(6 D_{P}\right)^{-1}=4.6 \pm 0.5 \mathrm{ps},\left(k_{P-W}\right)^{-1}=9 \pm 1 \mathrm{ps},\left(k_{W-P}\right)^{-1}=18 \pm 2 \mathrm{ps}$, and a total exchange rate of $\tau_{e x}=\left(k_{P-W}+k_{W-P}\right)^{-1}=6 \pm 1 \mathrm{ps}$, consistent with our previous result (4). We also fit the $T_{W}$-dependent $I_{\text {iso }}^{(i j)}$ and $I_{\text {aniso }}^{(i j)}$ peak volumes, as shown in Fig. 3. 
These fitting results agree with the response function global fitting, within experimental error. Within the angular jump-exchange kinetic model, only large values of $\Theta, D_{W}$, and $D_{P}$ could lead to the very small anisotropy of the cross-peak $I_{\text {aniso }}^{(W P)}$, but the anisotropy of the diagonal peaks greatly constrains $D_{W}$ and $D_{P}$. These constraints, as well as the high sensitivity of the cross-peak anisotropy to small changes in the jump angle around $49^{\circ}$, render the $\Theta$ value extracted from the measurements robust.

We also performed Car-Parrinello molecule dynamics (CPMD) simulations (25) of aqueous $6 \mathrm{M} \mathrm{NaClO}_{4}$ to complement our experimental studies (20). The CPMD methodology differs substantially from that used previously $(1,5,14)$ to study H-bond switching, yet as shown in Fig. 4, very similar angular jump dynamics emerge in the CPMD simulations of H-bond switching between $\mathrm{OD}_{\mathrm{P}}$ and $\mathrm{OD}_{\mathrm{W}}$ configurations $(1,5)$. Analysis of the mean trajectory indicates that the orientation changes on two time scales, with a $40^{\circ}$ jump occurring with a 50 fs time constant and a $27^{\circ}$ reorientation occurring with a 1 ps time constant. By simulating the same solution as that studied experimentally, we can make a direct comparison between experiment and simulation. The results of the simulation qualitatively agree with our experimental results, validating the angular jump mechanism for $\mathrm{H}$-bond switching in aqueous $\mathrm{NaClO}_{4}$ solutions. The time constant for the fast component resembles the period of a water librational motion, consistent with the assessment that the orientational jump reflects a primarily concerted rotational motion. The 1 ps process occurs too slowly to be viewed as concerted, but our experiment cannot accurately measure the detailed time evolution of the angular jump because the crosspeak intensity grows in with $\tau_{e x}=6 \mathrm{ps}$. Nonetheless, for $T_{W} \leq 2 \mathrm{ps}$ the cross-peak shows a very small anisotropy (Fig. S8) which has been accurately modeled with an impulsive 
angular jump and conforms to the predominantly sub-picosecond angular dynamics observed in the simulation. Whether the lack of quantitative agreement between experiment and simulation reflects limitations in the CPMD simulation or the modeling of the experimental data awaits further investigation.

\section{References and Notes}

1. D. Laage, J. T. Hynes, Proc. Natl. Acad. Sci. U.S.A 104, 11167 (2007).

2. C. J. Fecko, J. D. Eaves, J. J. Loparo, A. Tokmakoff, P. L. Geissler, Science 301, $1698(2003)$.

3. D. E. Moilanen, D. Wong, D. E. Rosenfeld, E. E. Fenn, M. D. Fayer, Proc. Natl. Acad. Sci. U.S.A 106, 375 (2009).

4. $\quad$ S. Park, M. Odelius, K. J. Gaffney, J. Phys. Chem. B 113, 7825 (2009).

5. D. Laage, J. T. Hynes, Science 311, 832 (2006).

6. Y. L. A. Rezus, H. J. Bakker, J. Chem. Phys. 123, 114502 (2005).

7. H. J. Bakker, Chem. Rev. 108, 1456 (2008).

8. $\quad$ C. P. Lawrence, J. L. Skinner, J. Chem. Phys. 118, 264 (2003).

9. J. B. Asbury et al., J. Phys. Chem. A 108, 1107 (Feb, 2004).

10. J. D. Eaves et al., Proc. Natl. Acad. Sci. U.S.A 102, 13019 (2005).

11. M. L. Cowan et al., Nature 434, 199 (Mar, 2005).

12. B. Nigro, S. Re, D. Laage, R. Rey, J. T. Hynes, J. Phys. Chem. A 110, 11237 (2006).

13. A. Luzar, D. Chandler, Phys. Rev. Lett. 76, 928 (1996).

14. R. Ludwig, ChemPhysChem 8, 44 (2007).

15. J. F. Cahoon, K. R. Sawyer, J. P. Schlegel, C. B. Harris, Science 319, 1820 (Mar 28, 2008).

16. J. Zheng et al., Science 309, 1338 (2005).

17. J. Zheng, K. Kwak, J. Xie, M. D. Fayer, Science 313, 1951 (2006).

18. S. Woutersen, Y. Mu, G. Stock, P. Hamm, Chem. Phys. 266, 137 (2001).

19. Y. S. Kim, R. M. Hochstrasser, Proc. Natl. Acad. Sci. U.S.A 102, 11185 (2005).

20. Methods are detailed in supporting online material at Science Online.

21. O. Golonzka, A. Tokmakoff, J. Chem. Phys. 115, 297 (2001).

22. M. T. Zanni, N. H. Ge, Y. S. Kim, R. M. Hochstrasser, Proc. Natl. Acad. Sci. U. S. A. 98, 11265 (2001).

23. K. Kwak, J. Zheng, H. Cang, M. D. Fayer, J. Phys. Chem. B 110, 19998 (2006).

24. A. Tokmakoff, J. Chem. Phys. 105, 1 (1996).

25. R. Car, M. Parrinello, Phys. Rev. Lett. 55, 2471 (1985).

26. M. J. and K. J. G. were supported through the PULSE Institute at the SLAC National Accelerator Laboratory by the U.S. Department of Energy Office of Basic Energy Sciences. M. O. thanks the Swedish Research Council (VR) for support and gratefully acknowledges generous grants of computer time at the Swedish National Supercomputer Center (NSC) and Center for Parallel Computing (PDC). 
Fig. 1 Interpretation of polarization-selective 2DIR signals. The H-bond configurations appear schematically with the green spheres representing perchlorate ions. (A) Linearly polarized excitation pulses selectively label vibrational dipoles oriented parallel to the $z$ direction, as shown in the diagonal regions of the $2 \mathrm{D}$ spectra. After a $T_{W}$ waiting time, vibrationally labeled hydroxyl groups that have not changed H-bond configuration contribute to the diagonal component of the 2DIR signal with minimal reorientation of the OD transition dipole. Vibrationally labeled hydroxyl groups that have undergone Hbond exchange generate cross-peak spectral intensity. If the exchange proceeds through large angular jumps, the hydroxyl groups associated with the cross-peaks will show markedly different polarization dependence than those resonating along the diagonal. This can be seen most clearly by comparing the ratio of cross-peak and diagonal-peak intensities generated with (B) all parallel laser polarizations $\left(S_{z z z z}, E_{z}^{e x c}, E_{z}^{e m i}\right)$ and (C) cross-polarized laser pulses $\left(S_{z z y y}, E_{z}^{e x c}, E_{y}^{e m i}\right)$. (D) FTIR absorption spectrum for $6 \mathrm{M}$ $\mathrm{NaClO}_{4}$ dissolved in $\mathrm{H}_{2} \mathrm{O}$ containing 5\% HOD. The lower frequency peak at $2534 \mathrm{~cm}^{-1}$ corresponds to the $\mathrm{OD}$ stretch donating a $\mathrm{H}$-bond to other water molecules $\left(\mathrm{OD}_{\mathrm{W}}\right)$; the peak at $2633 \mathrm{~cm}^{-1}$ corresponds to the OD stretch donating a H-bond to a perchlorate ion $\left(\mathrm{OD}_{\mathrm{P}}\right)$

Fig. 2 Normalized Polarization-selective 2DIR spectra at $T_{W}=0.2,3$ and 7ps. (A) Parallel polarization spectra $\left(S_{z z z z}\right),\left(\right.$ B) perpendicular polarization spectra, $\left(S_{z z y y}\right)$, and (C) anisotropic spectra, $\left(S_{\text {aniso }}=S_{z z z z}-S_{z z y y}\right)$. Peak assignments can be found in the text. The cross-peaks show very little anisotropy compared to the diagonal peaks $(\mathrm{C})$, clearly 
indicating that large angle rotation of hydroxyl groups accompany H-bond switching. (D) Calculated anisotropic spectra based on equations (1) and (2).

Fig. 3 Logarithmic plot of the polarization-selective peak volumes for the isotropic $I_{i s o}^{(i j)}=I_{z z z z}^{(i j)}+2 I_{z z y y}^{(i j)}$, and anisotropic, $I_{\text {aniso }}^{(i j)}=I_{z z z z}^{(i j)}-I_{z z y y}^{(i j)}$, signals for the $i j=P P$ diagonalpeak and the $i j=W P$ cross-peak. The solid lines give the kinetic model fit to the data with a H-bond exchange rate of $6 \pm 1 \mathrm{ps}$ and a jump angle of $49 \pm 4^{\circ}$.

Fig. 4 CPMD simulation of the jump angle for H-bond exchange between the $\mathrm{OD}_{\mathrm{P}}$ and the $\mathrm{OD}_{\mathrm{W}}$ configurations for aqueous $6 \mathrm{M} \mathrm{NaClO}_{4}$ solution. The data have been fit with a sum of two error functions (solid line). Results show the change in angle proceeding with two time constants, $50 \mathrm{fs}$ for an initial $40^{\circ}$ angular jump and $1 \mathrm{ps}$ for a slower $27^{\circ}$ angular rotation. 
Fig. 1

(A)

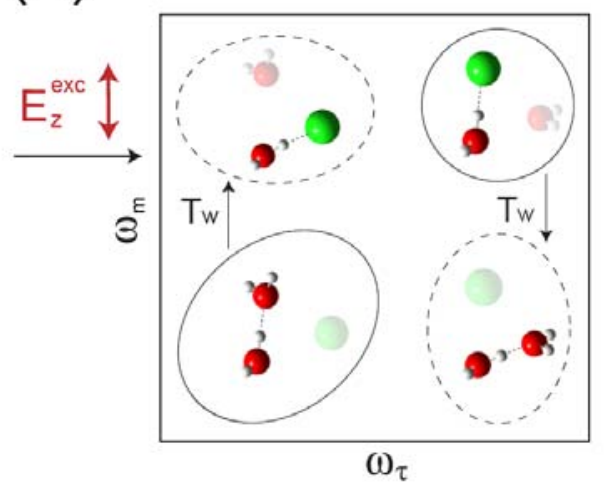

(C) $S_{z x y} \quad \downarrow \leftrightarrow E_{y}^{e m i}$

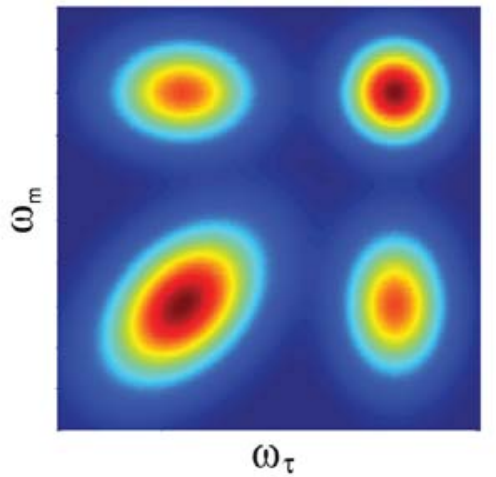

(B) $\mathrm{S}_{2 z z z}$

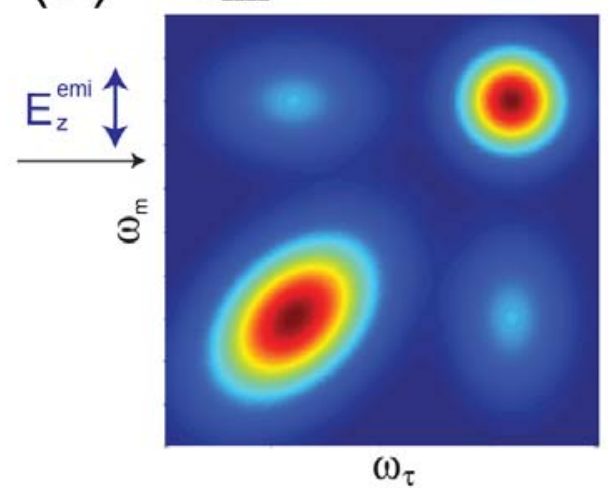

(D)

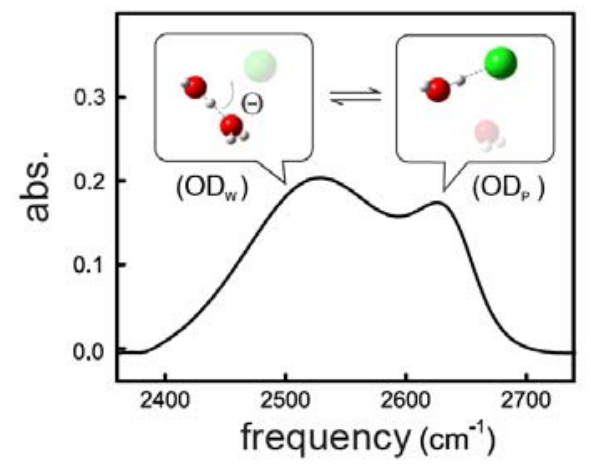


Fig. 2

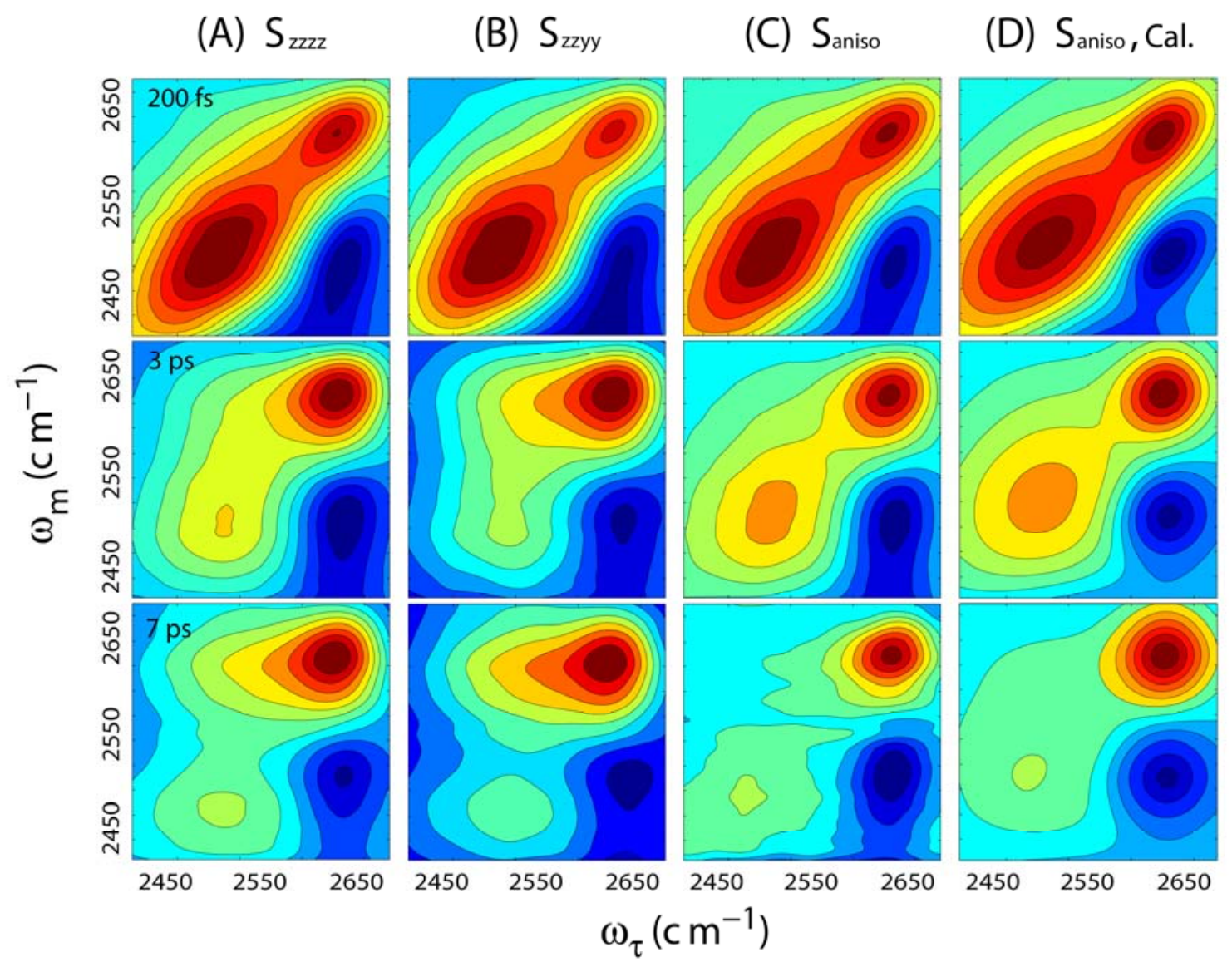


Fig. 3

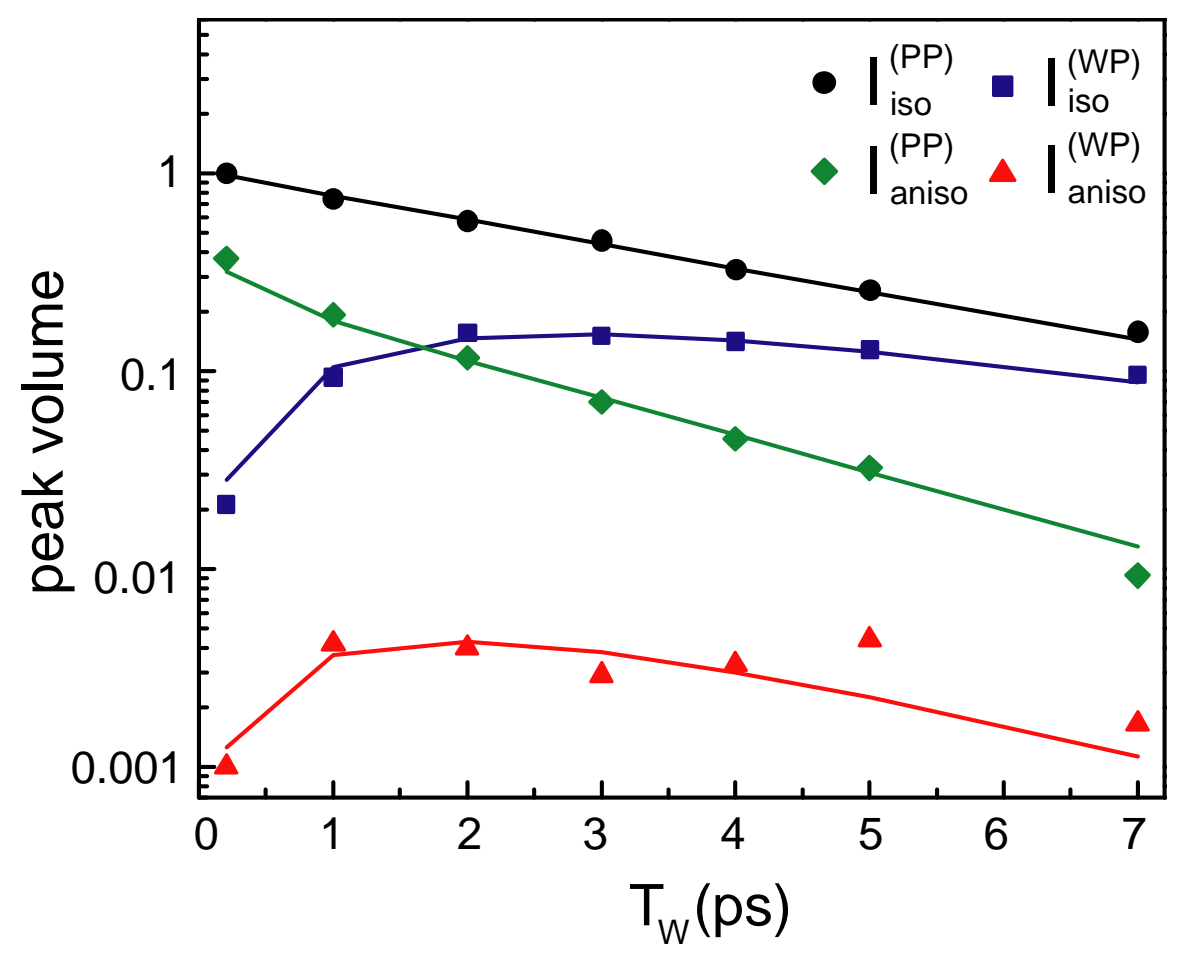


Fig. 4

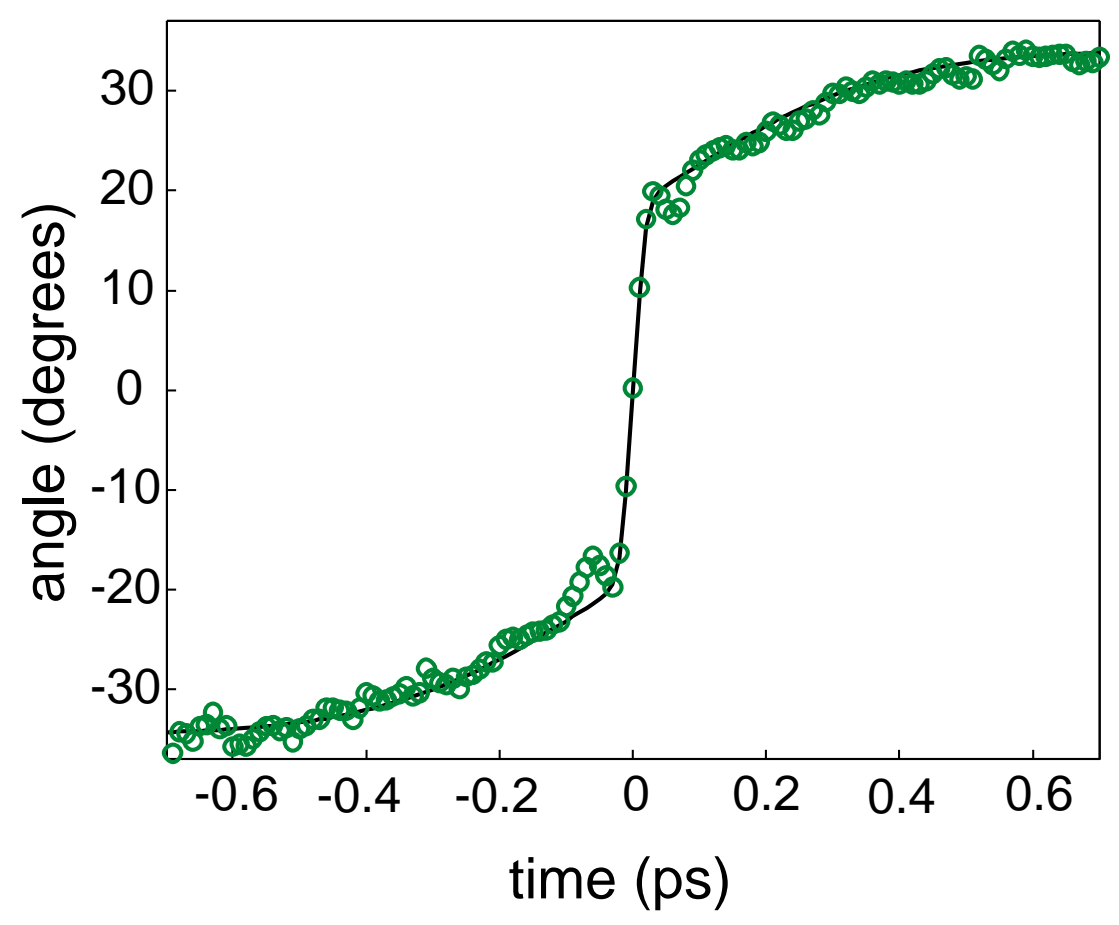




\section{Supporting Online Material}

\begin{tabular}{|l|l|l|}
\hline & Experimental Design and Methods & Page 2 \\
\hline & Variants of Kinetic Models & Page 3-7 \\
\hline & CPMD Simulations & Page 8-10 \\
\hline Fig. S1-S2 & 2DIR Setup and Phasing Method & Page 11-12 \\
\hline Fig. S3-S6 & Polarization Dependent 2DIR Data & Page 13-16 \\
\hline Fig. S7-S8 & Anisotropy Data and Analysis & Page 17-18 \\
\hline Fig. S9-S11 & CPMD Simulation Results & Page 19-21 \\
\hline Table S1 & Best-Fit Parameters & Page 22 \\
\hline & References & Page 23 \\
\hline
\end{tabular}




\section{DIR Experimental Design and Implementation}

The experimental set-up is shown schematically in Fig. S1. The collection of polarization resolved 2DIR spectra presents two primary technical challenges: the relative time delays between laser pulses must be determined for accurate phasing of the experimental data and the relative polarization and power of the four laser pulses used in the experiment must be accurately controlled. This section will describe the methods we used to control these parameters, as well as describe why our particular experiment and the conclusions we draw from the experiment are not as sensitive to the subtle details of the experimental set-up as previous attempts to utilize 2DIR to measure spectral diffusion dynamics or polarization resolved pump-probe to measure orientational dynamics slower than the population relaxation and solvent heating.

We use the pump-probe projection method to set the relative time delays (phases) between the first and second and the third and fourth laser pulses in the 2DIR measurement (Fig. S2). This method was developed by Tokmakoff and co-workers (S1) and a detailed description of the implementation can be found in Park et. al (S2). Subtle errors in phasing will have minimal impact on the data and analysis presented in this study because peak volumes are relatively insensitive to the phase, unlike peak shapes.

Anisotropy measurements often involve the measurement of small differences between two signals, making them sensitive to systematic errors in the laser polarization and power that can result from polarization dependent reflectivities of optical components. Additionally, polarization resolved pump-probe measurements of the water hydroxyl stretch anisotropy have been further limited by orientational relaxation times comparable to or longer than the excited state relaxation time constants and the influence of thermally generated transients that obscure the desired pump-probe signal at larger time delays (S3). Studying a concentrated aqueous ionic solution, however, greatly reduces these complications by increasing the excited state lifetime ( $\tau=2.54 \mathrm{ps}$ for $\mathrm{OD}_{\mathrm{W}}$ and $\tau=5.24 \mathrm{ps}$ for $\mathrm{OD}_{\mathrm{P}}$, compared to $\tau=1.8 \mathrm{ps}$ for $\mathrm{OD}$ in isotopically mixed water) and significantly reducing the magnitude of the thermal transient and the rate of heating.

Most importantly, the jump angle extracted from the experiment proves to be much more robustly measured than the slow orientational relaxation in a polarization resolved pump-probe measurement. This occurs for two primary reasons. Firstly, the jump angle measures the relative anisotropy of the diagonal peaks compared to the off-diagonal peaks. Consequently, the measurement is most sensitive to the jump angle for $T_{W} \leq 3 \mathrm{ps}$, time delays where significant anisotropic population persists for the diagonal peak intensities in the 2DIR spectra. Secondly, for the very small cross-peak anisotropies measured in the spectra, relative to the diagonal peak anisotropies, a jump angle of roughly $49^{\circ}$ must be utilized. Small changes in the $49^{\circ}$ jump angle lead to large changes in cross-peak anisotropy. This makes the jump angle extracted from the measurement insensitive to small experimental errors and the details of the experimental modeling. This will be discussed in greater detail in the modeling section of the SOM. 


\section{Jump-Exchange Kinetic (JEK) Model}

The Jump-Exchange Kinetic (JEK) model, with a jump angle $\Theta$, involves the solution of the following differential equations:

$$
\begin{aligned}
& \frac{d}{d t}\left[N_{W}(t) f_{W}(\Omega, t)\right]=-\left(K_{W}+K_{W-P}+D_{W} \hat{I}^{2}\right)\left[N_{W}(t) f_{W}(\Omega, t)\right]+\frac{K_{P-W}}{2 \pi} \int d \Omega^{\prime} \delta\left(\hat{\mu} \cdot \hat{\mu}^{\prime}-\cos \Theta\right)\left[N_{P}(t) f_{P}\left(\Omega^{\prime}, t\right)\right] \\
& \frac{d}{d t}\left[N_{P}(t) f_{P}(\Omega, t)\right]=-\left(K_{P}+K_{P-W}+D_{P} \hat{I}^{2}\right)\left[N_{P}(t) f_{P}(\Omega, t)\right]+\frac{K_{W-P}}{2 \pi} \int d \Omega^{\prime} \delta\left(\hat{\mu} \cdot \hat{\mu}^{\prime}-\cos \Theta\right)\left[N_{W}(t) f_{W}\left(\Omega^{\prime}, t\right)\right]
\end{aligned}
$$

Where $\hat{\mu}$ is the unitary vector of the dipole orientation in $\Omega$ and the Dirac $\delta$ function forces the jump angle to equal $\Theta .-\hat{I}^{2}=\frac{1}{\sin \theta} \frac{\partial}{\partial \theta}\left(\sin \theta \frac{\partial}{\partial \theta}\right)+\frac{1}{\sin ^{2} \theta} \frac{\partial^{2}}{\partial \phi^{2}}$ is the angular diffusion operator, having spherical harmonic eigenfunctions. We insert a spherical harmonic expansion, $f(\Omega, t)=\sum_{l, m} C_{l m}(t) Y_{l m}(\Omega) \int d \Omega_{0} Y_{l m}^{*}\left(\Omega_{0}\right) f\left(\Omega_{0}\right) \quad$ into differential equations (1) and (2), and apply the following initial conditions for the angular probability distribution function, $f_{i}\left(\Omega_{0}\right)=3 \cos ^{2} \theta=2 \sqrt{\frac{4 \pi}{5}} Y_{20}\left(\Omega_{0}\right)+\sqrt{4 \pi} Y_{00}\left(\Omega_{0}\right)$. We can remove the $\Omega$ variable and reduce the above differential equations to equations of $\left[N_{i}(t) C_{l}(t)\right]$, and solve them analytically. Based on the initial conditions, only $l=0,2$ generate non-vanishing terms. To solve the integral, $\frac{1}{2 \pi} \iint d \Omega d \Omega^{\prime} Y_{l 0}(\Omega) Y_{l 0}\left(\Omega^{\prime}\right) \delta\left(\hat{\mu} \cdot \hat{\mu}^{\prime}-\cos \Theta\right)$, we apply the Wigner D-matrix for the rotation of spherical harmonics. The integral yields $P_{l}\left(\hat{\mu} \cdot \hat{\mu}^{\prime}\right)=P_{l}(\cos \Theta)$. And the only terms left are: $P_{0}(\cos \Theta)=1, P_{2}(\cos \Theta)=\left(3 \cos ^{2} \Theta-1\right) / 2$.

The above derivation uses a Green's function methodology for a single jump angle. For a distribution of jump angle $\zeta(\Theta)$, we replace the $\delta\left(\hat{\mu} \cdot \hat{\mu}^{\prime}-\cos \Theta\right)$ function with $\int d \Theta \zeta(\Theta) \delta\left(\hat{\mu} \cdot \hat{\mu}^{\prime}-\cos \Theta\right)$ in equations (1) and (2). Now the integral becomes:

$\frac{1}{2 \pi} \iint d \Omega d \Omega^{\prime} Y_{l 0}(\Omega) Y_{l 0}\left(\Omega^{\prime}\right) \int d \Theta \zeta(\Theta) \delta\left(\hat{\mu} \cdot \hat{\mu}^{\prime}-\cos \Theta\right)=\int d \Theta \zeta(\Theta) P_{l}(\cos \Theta)=\left\langle P_{l}(\cos \Theta)\right\rangle$

Notice $\left\langle P_{0}(\cos \Theta)\right\rangle=P_{0}(\cos \langle\Theta\rangle) \equiv 1$, but usually $\left\langle P_{2}(\cos \Theta)\right\rangle \neq P_{2}(\cos \langle\Theta\rangle)$. The implication of this inequality will be discussed below.

Ensemble averaging for different laser polarizations gives $\left(N_{i}(t)\right)_{z z z z}=\int d \Omega\left[N_{i}(t) f_{i}(\Omega, t)\right] \cos ^{2} \theta$. And $\left(N_{i}(t)\right)_{z z y y}=\int d \Omega\left[N_{i}(t) f_{i}(\Omega, t)\right] \sin ^{2} \theta \cos ^{2} \phi$ With a final result of

$$
\left(\begin{array}{l}
N_{W}\left(T_{w}\right) \\
N_{P}\left(T_{w}\right)
\end{array}\right)_{z z z z}=\left(e^{-A \cdot T_{w}}+\frac{4}{5} e^{-B \cdot T_{w}}\right) \times \frac{1}{3}\left(\begin{array}{l}
N_{W}(0) \\
N_{P}(0)
\end{array}\right),\left(\begin{array}{l}
N_{W}\left(T_{w}\right) \\
N_{P}\left(T_{w}\right)
\end{array}\right)_{z z y y}=\left(e^{-A \cdot T_{w}}-\frac{2}{5} e^{-B \cdot T_{w}}\right) \times \frac{1}{3}\left(\begin{array}{l}
N_{W}(0) \\
N_{P}(0)
\end{array}\right)
$$

where, $A=\left(\begin{array}{cc}k_{W}+k_{W-P} & -k_{P-W} \\ -k_{W-P} & k_{P}+k_{P-W}\end{array}\right)$ and $B=\left(\begin{array}{cc}k_{W}+k_{W-P}+6 D_{W} & -\left\langle P_{2}(\cos \Theta)\right\rangle k_{P-W} \\ -\left\langle P_{2}(\cos \Theta)\right\rangle k_{W-P} & k_{P}+k_{P-W}+6 D_{P}\end{array}\right)$.

The isotropic and anisotropic contributions are

$$
\left(\begin{array}{l}
N_{W}\left(T_{w}\right) \\
N_{P}\left(T_{w}\right)
\end{array}\right)_{\text {iso }}=e^{-A \cdot T_{w}} \times\left(\begin{array}{c}
N_{W}(0) \\
N_{P}(0)
\end{array}\right) \text { and }\left(\begin{array}{l}
N_{W}\left(T_{w}\right) \\
N_{P}\left(T_{w}\right)
\end{array}\right)_{\text {aniso }}=\frac{2}{5} e^{-B \cdot T_{w}} \times \frac{1}{3}\left(\begin{array}{c}
N_{W}(0) \\
N_{P}(0)
\end{array}\right)
$$


We use (4) in the response function calculation to fit the whole 2D data set (Fig. S3-S5) and the polarization dependent pump-probe data, and (5) is used to fit the extracted peak volumes (Fig. S6) for the $I_{i s o}$ and $I_{\text {aniso }}$ signals.

$\left\langle P_{2}(\cos \Theta)\right\rangle$ is the experimental observable we've measured. We seemingly cannot determine $\langle\Theta\rangle$ without knowing the character of the distribution $\zeta(\Theta)$. However, if we assume a relatively symmetric distribution for $\zeta(\Theta)$, consistent with the CPMD distribution shown in Fig. S9, we can extract $\langle\Theta\rangle$ from the measurement. Notice $P_{2}(\cos \Theta)$ is an odd function about $\Theta=45^{\circ}$ with an offset of $P_{2}\left(\cos 45^{\circ}\right)$, thus the symmetry point at $\Theta=45^{\circ}$ has $\left\langle P_{2}(\cos \Theta)\right\rangle=P_{2}(\cos \langle\Theta\rangle)$ for a symmetric $\zeta(\Theta)$. For our measured jump angle of $\langle\Theta\rangle=49 \pm 4^{\circ}$, we have $\left\langle P_{2}(\cos \Theta)\right\rangle \approx P_{2}(\cos \langle\Theta\rangle)$. (Based on the width of the $\zeta(\Theta)$ distribution from the CPMD simulation shown in Fig. S9, we estimate $\zeta(\Theta)$ to have a Gaussian distribution with a FWHM of $30^{\circ}$. Centering this distribution at $\langle\Theta\rangle=49^{\circ}$, the mean angle extracted from $P_{2}^{-1}\left(\left\langle P_{2}(\cos \Theta)\right\rangle\right)=48.6^{\circ}$.)

Within this model, the intra-configurational orientational dynamics has been treated with a diffusion constant. This treatment does not support a particular mechanism for intra-configurational orientational memory loss. As shown by Laage and Hynes (S4), a single exponential decay of the anisotropy does not distinguish between angular jump induced loss of orientational memory or diffusive loss of orientational memory. We expect orientational relaxation within a H-bond configuration to also result from angular jumps associated with intra-configurational $\mathrm{H}$-bond switches. These $\mathrm{H}-$ bond switching events, however, cannot be directly observed with 2DIR because they do not cause the OD stretch frequency to switch between the two peaks in the vibrational absorption spectrum. 


\section{Embedding wobbling in a cone orientational dynamics into the JEK model}

While conceptually valuable, the orientational dynamics of water molecules that remain within a particular $\mathrm{H}$-bond configuration deviate from the single exponential decays generated with the JEK model. We have extended the JEK model to include wobbling in a cone bi-exponential orientational dynamics within each H-bond configuration, while leaving the angular jump dynamics associated with a H-bond configuration switch unchanged. This model provides an accurate treatment of the water orientational dynamics observed in multiple experiments $(S 5, S 6)$ and confirms that the jump angle extracted from the experimental data does not depend upon the details of the modeling.

The wobbling in a cone extension of the JEK model assumes that wobbling motion is independent of other motions, such as the chemical exchange. The wobbling model $(S 7$, S8) represents the time dependent dipole-dipole correlation function as a product of a wobbling term $C_{2}^{\text {mob }}(t)$ and an orientation diffusion term, $e^{-6 D_{o r} t}$ :

$$
C_{2}(t)=e^{-6 D_{o r} t} C_{2}^{w o b}(t)
$$

In order to embed wobbling in the kinetic differential equations, we parameterize (6) into a diffusion equation with an effective time-dependent diffusion rate $D_{\text {eff }}(t)$.

$$
\frac{d}{d t} C_{2}(t)=-6 D_{e f f}(t) C_{2}(t) \text {, with } 6 D_{e f f}(t)=-\frac{d}{d t} \ln \left[C_{2}(t)\right]=6 D_{o r}-\frac{d}{d t} \ln \left[C_{2}^{\text {wob }}(t)\right]
$$

Now we replace the diffusion constant in differential equations (1) and (2) with (7). Following the same procedures discussed previously, we get a result with the same form as (4), with the only change being in the B matrix, which is now time dependent:

$$
B^{\prime}\left(T_{w}\right)=\left(\begin{array}{cc}
K_{W}+K_{W-P}+6 D_{W}-\ln \left[C_{2 W}^{w o b}\left(T_{w}\right)\right] / T_{w} & -\left\langle P_{2}(\cos \Theta)\right\rangle K_{P-W} \\
-\left\langle P_{2}(\cos \Theta)\right\rangle K_{W-P} & K_{P}+K_{P-W}+6 D_{P}-\ln \left[C_{2 P}^{w o b}\left(T_{w}\right)\right] / T_{w}
\end{array}\right)
$$

The strict solution of the wobbling in a cone model is a sum over infinite number of exponentials $(S 7, S 8)$. Here we use an approximate result widely accepted in treating water orientational dynamics $(S 7, S 8), C_{2}^{\text {mob }}(t)=S^{2}+\left(1-S^{2}\right) e^{-t / \tau_{e f f}}$.

$\mathrm{S}$ and $\tau_{\text {eff }}$ are new fitting parameters included to fit both the 2DIR peak volumes and the pump-probe data. Particularly, the order parameter $S=\cos \theta_{c}\left(1+\cos \theta_{c}\right) / 2$, where $\theta_{c}$ is the cone semi-angle.

In order to fit the pump-probe data, we express the pump-probe signal as:

$$
\begin{aligned}
& \left(\begin{array}{c}
I_{W}(t) \\
I_{P}(t)
\end{array}\right)_{z z z z}=\left(\begin{array}{cc}
\left|\mu_{W}\right|^{2} & 0 \\
0 & \left|\mu_{P}\right|^{2}
\end{array}\right)\left(e^{-A \cdot t}+\frac{4}{5} e^{-B^{\prime}(t) \cdot t}\right)\left(\begin{array}{cc}
\left|\mu_{W}\right|^{2} & 0 \\
0 & \left|\mu_{P}\right|^{2}
\end{array}\right) \times \frac{1}{3}\left(\begin{array}{c}
N_{W}(0) \\
N_{P}(0)
\end{array}\right) \\
& \left(\begin{array}{c}
I_{W}(t) \\
I_{P}(t)
\end{array}\right)_{z z y y}=\left(\begin{array}{cc}
\left|\mu_{W}\right|^{2} & 0 \\
0 & \left|\mu_{P}\right|^{2}
\end{array}\right)\left(e^{-A \cdot t}-\frac{2}{5} e^{-B^{\prime}(t) \cdot t}\right)\left(\begin{array}{cc}
\left|\mu_{W}\right|^{2} & 0 \\
0 & \left|\mu_{P}\right|^{2}
\end{array}\right) \times \frac{1}{3}\left(\begin{array}{c}
N_{W}(0) \\
N_{P}(0)
\end{array}\right)
\end{aligned}
$$

With such an expression, the anisotropy dynamics measured with the polarization resolved pump-probe experiment can be fit with: 


$$
r_{i}(t)=\frac{I_{i}(t)_{z z z z}-I_{i}(t)_{z z y y}}{I_{i}(t)_{z z z z}+2 I_{i}(t)_{z z y y}}, i=W, P
$$

This analysis accounts for the fact that the pump-probe signal measures the orientational memory of the vibrationally excited water molecules within a particular H-bond configuration independent of those molecules configurational prior history. 


\section{Anisotropy dynamics from the different variants of the JEK model}

We calculate the anisotropy for each peak in the 2D spectra using equation (5):

$$
r^{i j}(t)=\frac{I_{\text {aniso }}^{i j}(t)}{I_{i s o}^{i j}(t)}=\frac{2}{5} \frac{\left[e^{-B \cdot t}\right]_{i j}}{\left[e^{-A \cdot t}\right]_{i j}}, i=W, P, j=W, P .
$$

We use this result and the different expressions for the $A$ and $B$ matrices generated by the various forms of the chemical exchange model to demonstrate the insensitivity of the jump angle to the details of the model.

1. The simple exchange model.

$$
\begin{aligned}
& r^{P P}(t)=\frac{2}{5} C_{2}^{P P}(\mathrm{t})=\frac{2}{5} e^{-\left(6 D_{W}+6 D_{P}\right) / 2} F^{P P}(t) \\
& r^{W P}(t)=\frac{2}{5} C_{2}^{W P}(\mathrm{t})=\frac{2}{5} e^{-\left(6 D_{W}+6 D_{P}\right) / 2} F^{W P}(t)
\end{aligned}
$$

$F^{i j}(t)$ are complicated functions of time and involve cosh and sinh functions of the $A$ and $B$ matrix elements, with $F^{i j}(0)=1$. Nonetheless, $F^{i j}(t)$ functions have sufficiently weak time dependence that they can be approximated as time independent to assist our qualitative discussion of the models (Fig. S7). We can see the anisotropy of both diagonal and cross-peaks orientationally relax with a rate dictated by a mean diffusion constant, and modified by the $F^{i j}(t)$ functions. This generates the same signal anisotropies for the diagonal and cross peaks, making the simple exchange model incompatible with the experimental data shown in Figs. S3-S6.

\section{The JEK model.}

$$
\begin{gathered}
r^{P P}(t)=\frac{2}{5} C_{2}^{P P}(\mathrm{t})=\frac{2}{5} e^{-\left(6 D_{W}+6 D_{P}\right) / 2} F_{j u m p}^{P P}(t) \\
r^{W P}(t)=\frac{2}{5}\left\langle P_{2}(\cos \Theta)\right\rangle C_{2}^{W P}(\mathrm{t})=\frac{2}{5}\left\langle P_{2}(\cos \Theta)\right\rangle e^{-\left(6 D_{W}+6 D_{P}\right) / 2} F_{j u m p}^{W P}(t)
\end{gathered}
$$

It's clearly seen that angular jump reduces the anisotropy of the cross-peak by a factor of $P_{2}(\cos \Theta)=\left(3 \cos \Theta^{2}-1\right) / 2$, while the diagonal peak anisotropy remains highly similar to the simple exchange model. For $\langle\Theta\rangle=50^{\circ}$ and a symmetric $\zeta(\Theta)$ distribution, $\left\langle P_{2}(\cos \Theta)\right\rangle \approx 0.12$, which is the source of the small $r^{W P}$.

\section{The wobbling in a cone corrected JEK model.}

$$
\begin{gathered}
r^{P P}(t)=\frac{2}{5} C_{2}^{P P}(\mathrm{t})=\frac{2}{5} \sqrt{C_{2 P}^{w o b}(t)} \sqrt{C_{2 W}^{w o b}(t)} e^{-\left(6 D_{W}+6 D_{P}\right) / 2} F_{\text {wob }}^{P P}(t) \\
r^{W P}(t)=\frac{2}{5}\left\langle P_{2}(\cos \Theta)\right\rangle C_{2}^{W P}(\mathrm{t})=\frac{2}{5}\left\langle P_{2}(\cos \Theta)\right\rangle \sqrt{C_{2 P}^{w o b}(t)} \sqrt{C_{2 W}^{w o b}(t)} e^{-\left(6 D_{W}+6 D_{P}\right) / 2} F_{\text {wob }}^{W P}(t)
\end{gathered}
$$

Note that wobbling has similar affects on the anisotropy of the diagonal and the crosspeak, and thus the wobbling model cannot explain our experimental data without the inclusion of a jump angle. This also demonstrates the insensitivity of the jump angle to the details of the orientational dynamics within the $\mathrm{OD}_{\mathrm{P}}$ and $\mathrm{OD}_{\mathrm{W}} \mathrm{H}$-bond configurations for jump angles consistent with our experimental results. 


\section{Ab initio Molecular Dynamics Simulation Methodology}

A more complete analysis of the molecular dynamics simulation can be found in Park et. al. (S9). Here we present the prior results most relevant to this paper. Ab initio CarParrinello molecular dynamics (CPMD) simulations (S10) were performed to characterize the structural and dynamical properties of aqueous $6 \mathrm{M} \mathrm{NaClO}_{4}$ solution. The CPMD simulations give an accurate description of many-body effects in concentrated aqueous ionic solutions, against which classical force fields can be evaluated, $(S 11, S 12)$ since CPMD calculates the forces on the fly with periodic density functional calculations using a gradient-corrected density functional, BLYP (S13, S14). However, since the computationally taxing CPMD methodology limits the system size to $7 \mathrm{Na}^{+}$and $7 \mathrm{ClO}_{4}{ }^{-}$ions dissolved in 46 water molecules and simulation duration to $20 \mathrm{ps,}$ we also performed 100 times longer duration and 8 times larger simulations with a classical force field (Parameters for the ions were taken from Heinje et al.(S11) and water is described by the flexible SPC force field(S15)) that confirmed the size and duration limits of the CPMD calculation do not distort the local structure and dynamics. The periodic density functional theory (DFT) calculations used pseudo-potentials in combination with an 85 Rydberg kinetic energy cut-off for the plane wave expansion of the Kohn-Sham wave-functions. For hydrogen, a local pseudo-potential parameterized with one Gaussian was used. The pseudo-potential for oxygen was of Troullier-Martins type $(S 16)$ expressed in the Kleinman-Bylander form, $(S 17)$ and were non-local in the $1=0$ channel. Goedecker pseudo-potentials were used for sodium and chlorine. $(S 18, S 19)$ We model the aqueous $6 \mathrm{M} \mathrm{NaClO}_{4}$ solution in the NVT ensemble with a $1.446 \mathrm{~g} / \mathrm{cm}^{3}$ density, and use a $0.1 \mathrm{fs}$ time-step and a fictitious electron mass of 500 a.u. We set the Nose-Hoover thermostat(S20-S22) to $350 \mathrm{~K}$ to compensate for the lack of a quantum treatment of the hydrogen atoms and the limitations of density functionals employed.(S23, S24) We initialized the 6 ps equilibration trajectory with the classical molecular dynamics (MD) simulation, and simulated two different deuterated systems. We used the simulation of $6 \mathrm{M} \mathrm{NaClO}_{4}$ with 1:45=DOH: $\mathrm{H}_{2} \mathrm{O}(20 \mathrm{ps})$ to study the H-bond structural and reorientational dynamics and the simulation of $6 \mathrm{M} \mathrm{NaClO}_{4}$ with $46 \mathrm{DOH}$ $(9 \mathrm{ps})$ to obtain the IR spectrum to get an appreciable OD component in the total electric dipole and to validate a local mode description of the hydroxyl stretch band. For reference, we also performed simulations of pure liquid water at $1.0 \mathrm{~g} / \mathrm{cm}^{3}$ and $350 \mathrm{~K}$ with either $64 \mathrm{DOH}(11 \mathrm{ps})$ or $64 \mathrm{H}_{2} \mathrm{O}(18 \mathrm{ps})$ molecules.

We use a standard geometric $\mathrm{H}$-bond criterion to analyze the H-bond structure: $R_{\mathrm{OO}}<$ $3.5 \AA, R_{\mathrm{HO}}<2.5 \AA$ and $\theta_{\mathrm{HOO}}<30^{\circ}$. Using this criterion, water molecules in aqueous $6 \mathrm{M}$ $\mathrm{NaClO}_{4}$ solution donate an average of $1.6 \mathrm{H}$-bonds. The simulations indicate that the solution consists of predominantly two different water species: (1) water molecules that donate two H-bonds to other water molecules ( $\mathrm{W}_{\mathrm{Ww}}, 34 \%$ ) and (2) water molecules that donate one $\mathrm{H}$-bond to a water molecule and the other $\mathrm{H}$-bond to a $\mathrm{ClO}_{4}{ }^{-}$ion $\left(\mathrm{W}_{\mathrm{WP}}, 27\right.$ $\%$ ). In addition, a large fraction of transient water species donate only a single $\mathrm{H}$-bond to either a water molecule $\left(\mathrm{W}_{\mathrm{WN}}, 21 \%\right)$ or a $\mathrm{ClO}_{4}{ }^{-}$ion $\left(\mathrm{W}_{\mathrm{NP}}, 9 \%\right)$. While short lived, these species show up in the instantaneous sampling of the structure of aqueous $6 \mathrm{M} \mathrm{NaClO}_{4}$ solution.

For the analysis of the H-bond exchange in the CPMD simulations of the $\mathrm{NaClO}_{4}$ solution, we have employed the tools developed by Laage and Hynes for pure water (S4). In the analysis we follow the same variables, but based on our H-bond definitions. 
Furthmore, H-bonds broken and reformed within 100 fs are considered intact from a spectroscopic viewpoint.

The simulation run contained roughly 500 switching events in each class of H-bond switches between and within the $\mathrm{OD}_{\mathrm{P}}$ and $\mathrm{OD}_{\mathrm{W}} \mathrm{H}$-bond configurations. The switches between the $\mathrm{OD}_{\mathrm{P}}$ and $\mathrm{OD}_{\mathrm{W}} \mathrm{H}$-bond configurations were used to generate the average angle jump dynamics presented in Fig. 4. Figure S9 presents the full distributions for the key structural degrees of freedom defining the H-bond and clearly shows that the angle has a well defined distribution before and after the H-bond exchange, where $\theta$ represents the angle between the direction of the OD group prior to and after $\mathrm{H}$-bond exchange, $\mathrm{O}$ represents the oxygen on the water molecule donating a H-bond, $\mathrm{X} 1$ represents the oxygen initially accepting the $\mathrm{H}$-bond, and $\mathrm{X} 2$ represents the oxygen that ends up accepting the H-bond. The resolution in the distributions would be improved by extended sampling, but the results are still clear.

\section{CPMD Simulations of the Linear IR Spectrum}

We calculate the IR spectra by Fourier transforming the total electric dipole time correlation function, $\mathbf{M}(t),(S 25-S 28)$

$$
\alpha(\omega) \cdot n(\omega)=\frac{4 \pi \omega \tanh (\beta \hbar \omega / 2)}{3 \hbar c V} \int_{-\infty}^{\infty} d t e^{-i \omega t}\langle\mathbf{M}(t) \cdot \mathbf{M}(0)\rangle,
$$

where $V$ is the volume, $T$ is the temperature, $\beta=1 / k T, n(\omega)$ is the refractive index, and $c$ is the speed of light in vacuum. In order to assign the two OD peaks of HOD in aqueous $6 \mathrm{M} \mathrm{NaClO}_{4}$ solution, we partition the CPMD simulation result into 1 ps time intervals and the OD spectra of HOD in different H-bond configurations are calculated in each time interval. Figure S10(A) displays the OD stretch spectra of HOD in different H-bond configurations. We have scaled the frequency axis so that the simulated gas phase vibrational frequency matches the experimental value. When compared with the OD stretch peaks in the experimental IR spectrum in Fig. S10(B), the peaks in the simulated IR spectrum are broader and the relative intensity of the high and low frequency OD peaks is different. While the CPMD simulation does not quantitatively reproduce the experimental FTIR spectrum, it clearly corroborates the assignment of the OD peak at $2534 \mathrm{~cm}^{-1}$ to $\mathrm{OD}_{\mathrm{W}}$ sub-ensemble and the $\mathrm{OD}$ peak at $2633 \mathrm{~cm}^{-1}$ to $\mathrm{OD}_{\mathrm{P}}$ sub-ensemble. The high frequency narrow peak results from the OD groups that do not donate a H-bond $\left(\mathrm{OD}_{\mathrm{N}}\right)$ or donate a $\mathrm{H}$-bonded to a $\mathrm{ClO}_{4}{ }^{-}$ion $\left(\mathrm{OD}_{\mathrm{P}}\right)$, while the low frequency broad peak results from $\mathrm{OD}$ groups that donate a $\mathrm{H}$-bond to another water molecule $\left(\mathrm{OD}_{\mathrm{W}}\right)$. It turns out that both $\mathrm{OD}_{\mathrm{N}}$ and $\mathrm{OD}_{\mathrm{P}}$ sub-ensembles contribute to the $\mathrm{OD}$ peak at the high frequency in the IR spectrum. However, the $\mathrm{OD}_{\mathrm{N}}$ sub-ensemble is short-lived and does not contribute to the $\mathrm{H}$-bond structural dynamics occurring on picosecond timescales observed in the CPMD simulations or ultrafast IR experiments.

\section{CPMD Simulations of Hydrogen Bond Dynamics in Aqueous $6 \mathrm{M} \mathrm{NaClO}_{4}$ Solution}

We calculate time correlation functions for both spectrally distinct hydroxyl stretch configurations $\left(\mathrm{OD}_{\mathrm{W}}\right.$ and $\left.\mathrm{OD}_{\mathrm{P}}\right)$ and structurally distinct molecular configurations $\left(\mathrm{W}_{\mathrm{WW}}\right.$ and $\mathrm{W}_{\mathrm{WP}}$ ). The $\mathrm{H}$-bond exchange dynamics between $\mathrm{OD}_{\mathrm{W}}$ and $\mathrm{OD}_{\mathrm{P}}$ can be extracted by calculating the time cross-correlation function (TCCF), $(S 29, S 4)$ 


$$
G_{\mathrm{P}-\mathrm{W}}^{\mathrm{TCCF}}(t)=1-\frac{\left\langle n_{\mathrm{P}}(0) n_{\mathrm{W}}(t)\right\rangle}{\left\langle n_{\mathrm{P}}\right\rangle\left\langle n_{\mathrm{W}}\right\rangle},
$$

where $n_{\mathrm{P}}$ is 1 when $\mathrm{OD}$ is $\mathrm{H}$-bonded to $\mathrm{ClO}_{4}{ }^{-}$ions or not $\mathrm{H}$-bonded and 0 otherwise, and $n_{\mathrm{W}}$ is 1 when $\mathrm{OD}$ is H-bonded to other water molecules and 0 otherwise. Here, $G_{\mathrm{P}-\mathrm{W}}^{\mathrm{TCCF}}(t)$ represents the $\mathrm{H}$-bond exchange dynamics between $\mathrm{OD}_{\mathrm{P}}$ and $\mathrm{OD}_{\mathrm{W}}$ sub-ensembles. The fluctuation-dissipation theorem predicts $G_{\mathrm{P}-\mathrm{W}}^{\mathrm{TCF}}(t) \cong \exp \left(-k_{\mathrm{ex}} t\right)$, with $k_{\mathrm{ex}}=k_{\mathrm{P}-\mathrm{W}}+k_{\mathrm{W}-\mathrm{P}}(S 30)$. Here, the simulated H-bond exchange time, $\tau_{e x}=\left(k_{\mathrm{ex}}\right)^{-1}$, corresponds to the slow exponential decay component of $G_{\mathrm{P}-\mathrm{W}}^{\mathrm{TCCF}}(t)$. In addition, the Hbond lifetime correlation function of each individual species can be obtained by calculating the TACF. For example, the H-bond lifetime TACF of $\mathrm{OD}_{\mathrm{W}}$ sub-ensemble equals.

$$
G_{\mathrm{W}}^{\mathrm{TACF}}(t)=\frac{\left\langle n_{\mathrm{W}}(0) n_{\mathrm{W}}(t)\right\rangle-\left\langle n_{\mathrm{W}}\right\rangle^{2}}{\left\langle n_{\mathrm{W}}^{2}\right\rangle-\left\langle n_{\mathrm{W}}\right\rangle^{2}} .
$$

This TACF represents the configurational memory of a water molecule that initially resides in the $\mathrm{OD}_{\mathrm{W}}$ configuration before switching to the $\mathrm{OD}_{\mathrm{P}}$ configuration. 


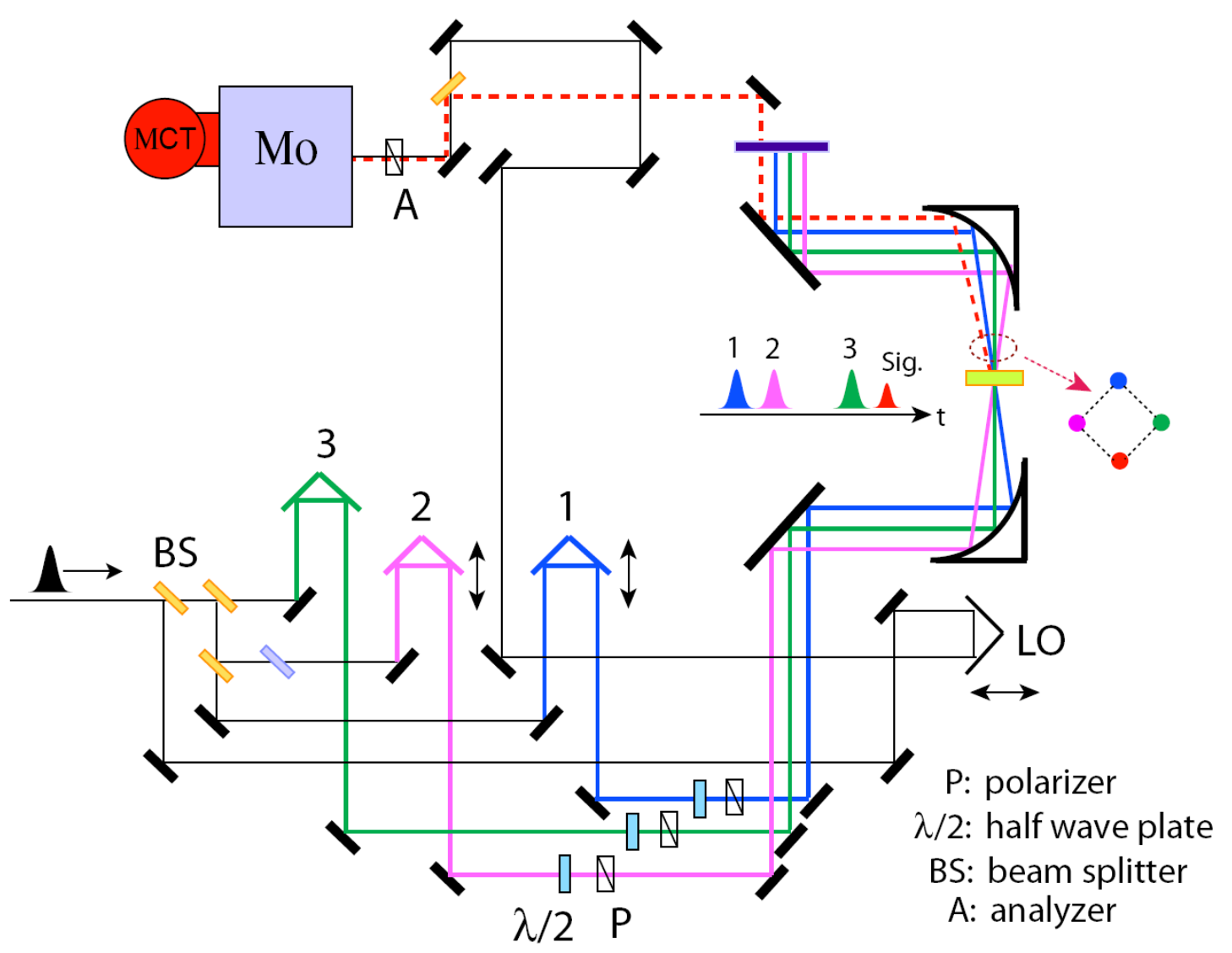

Fig. S1. Experimental setup. Time zero and spatial overlap of the three pulses at the sample position are optimized with a Frequency Resolved Optical Gating (FROG) measurement using the non-resonant response of $\mathrm{CCl}_{4}$. Chirp is minimized by adjusting the thickness of $\mathrm{CaF}_{2}$ plates inserted in the beam. We optimize the time delay between pulses 1 and 2 and pulses 3 and 4 with the pump-probe projection method (S2).The polarization of each beam is controlled by a half wave plate and polarizer, with extinction ratio of 1:200. Beam 3 is fixed at a $z$ polarization, while beam 1 and 2 are set to both $z$ or both $y$ polarization, generating an $S_{z z z z}$ or $S_{z z y y}$ signal. An analyzer polarized in the $z$ direction is placed before the detector. Changing the polarization of beams 1 and 2 results in negligible change in the pump beam power at the sample. 


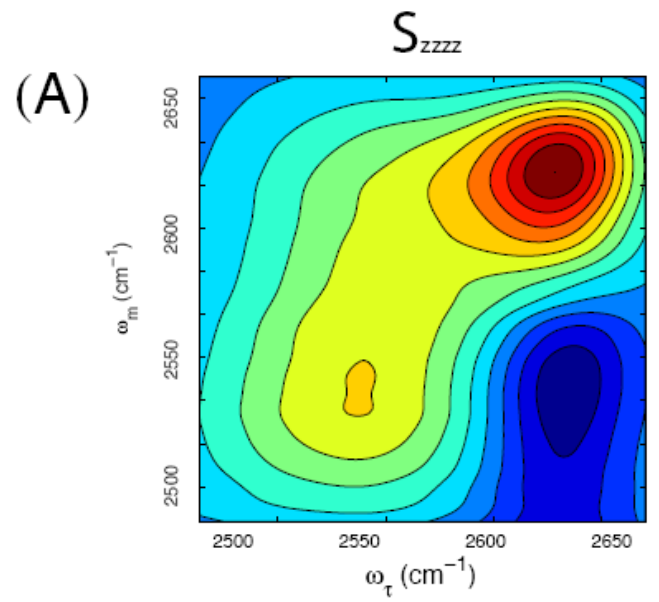

(B)
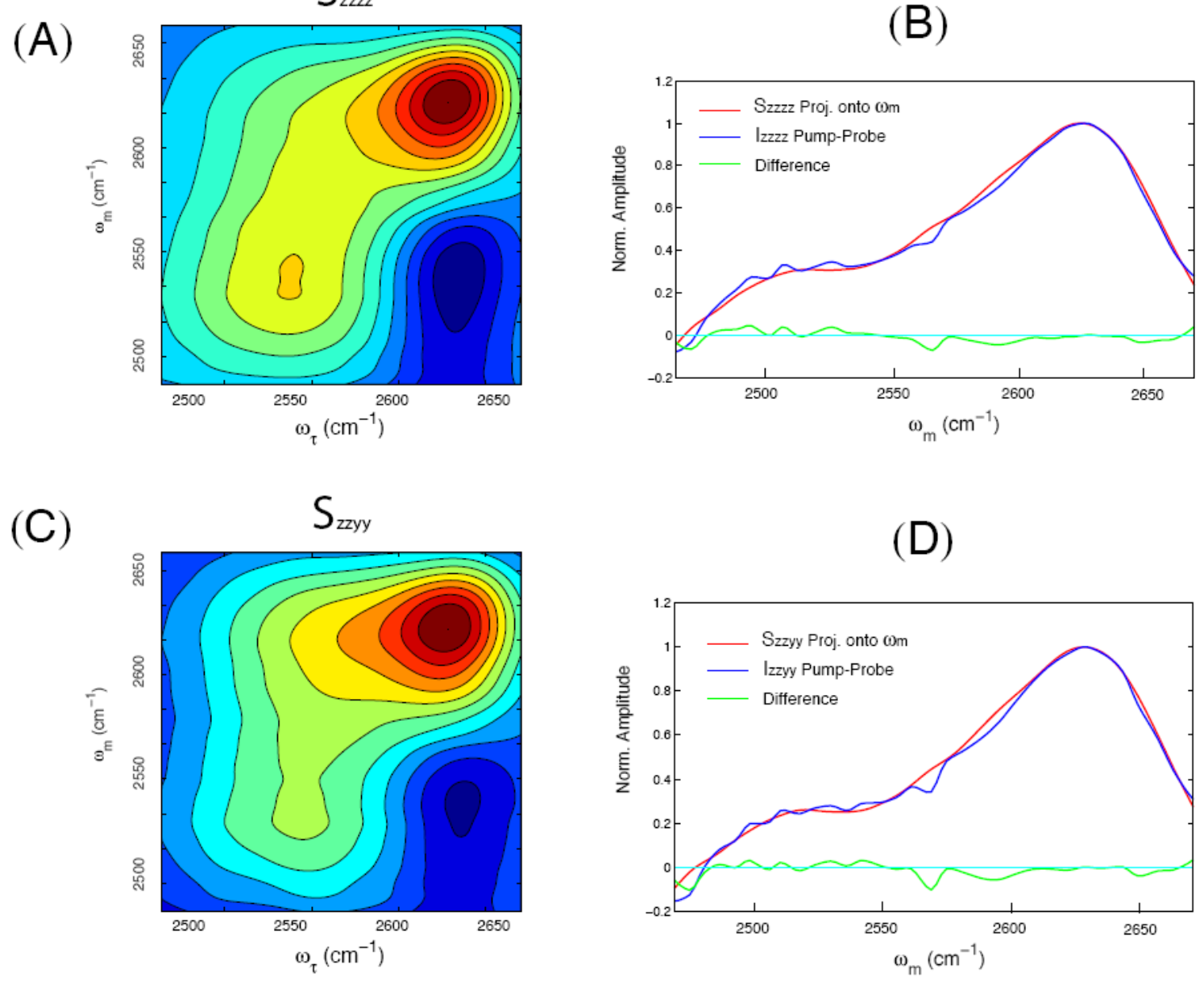

Fig. S2 Depiction of the method used to phase the 2D spectra. The parallel 2DIR spectra $S_{z z z z}$ (A) are projected onto $\omega_{m}$ (an integration over $\omega_{\tau}$ ). This projected spectra is adjusted to match the parallel Pump-Probe spectra at the same time delay (B). While the $\omega_{m}$ projection of perpendicular 2D spectra (C) is adjusted to fit the perpendicular PumpProbe spectra(D). The parameters and detailed method of phasing 2DIR data can be found in the paper of Park et. al. (S2). The spectra shown in Fig. S2 all correspond to $T_{W}=3 \mathrm{ps}$. 


\section{$\mathrm{S}_{z z z z}$ : Parallel}

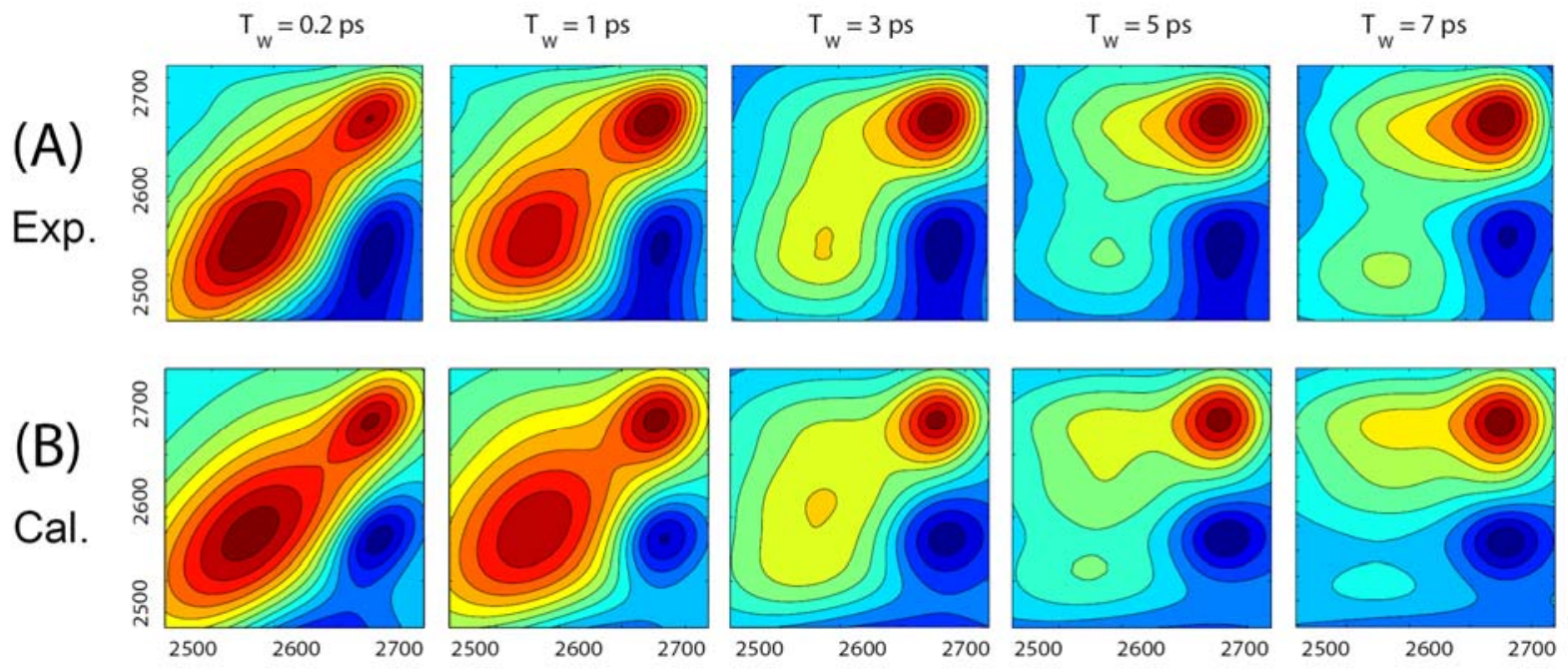

Fig. S3 The parallel geometry $S_{z z z z}$ experimental 2D spectra (A) and the calculated spectra (B) using the response function and jump exchange kinetic model fitting results. 


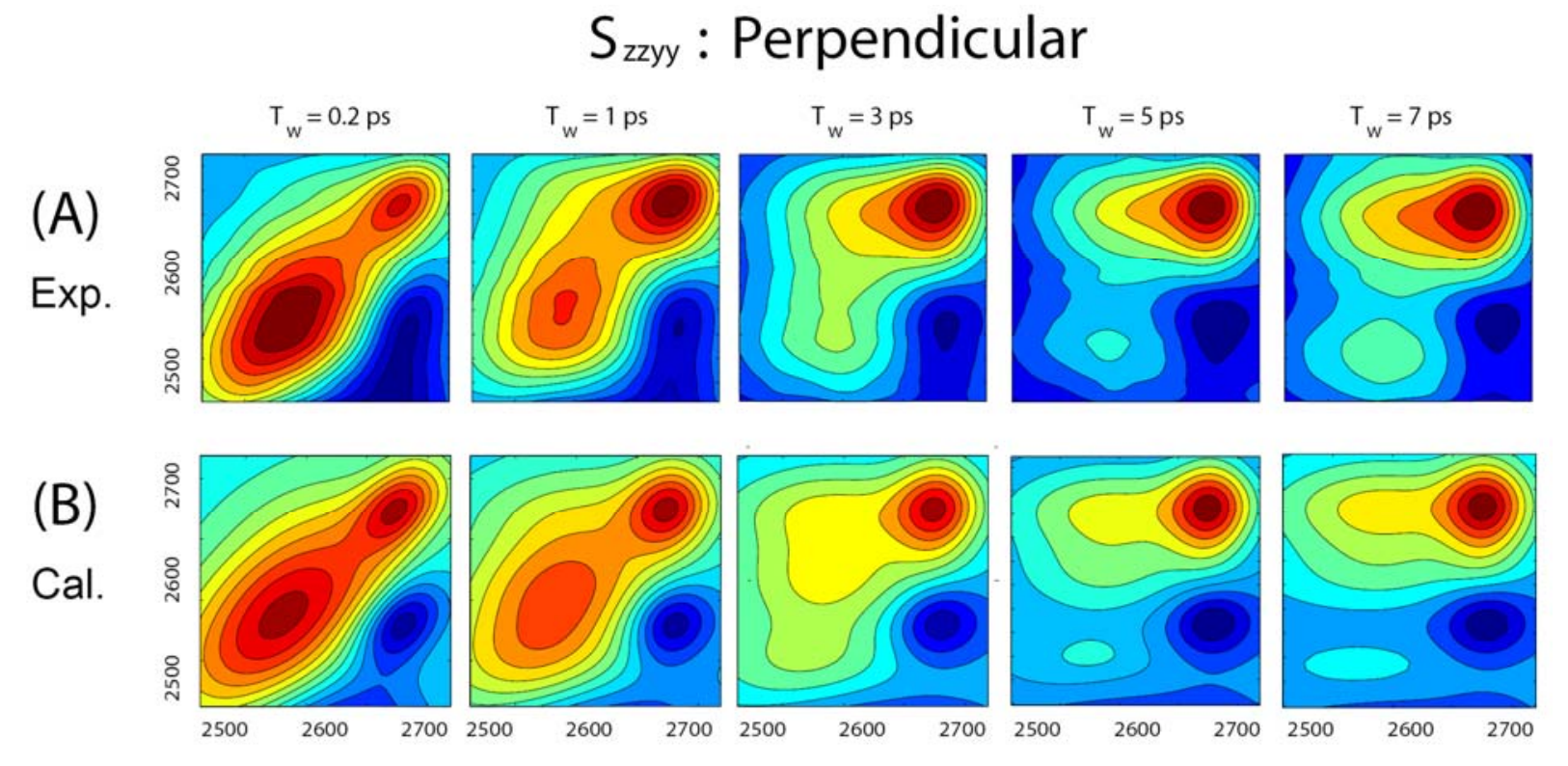

Fig. S4 The perpendicular geometry $S_{z z y y}$ experimental 2D spectra (A) and the calculated spectra (B) using the response function and jump exchange kinetic model fitting results. 


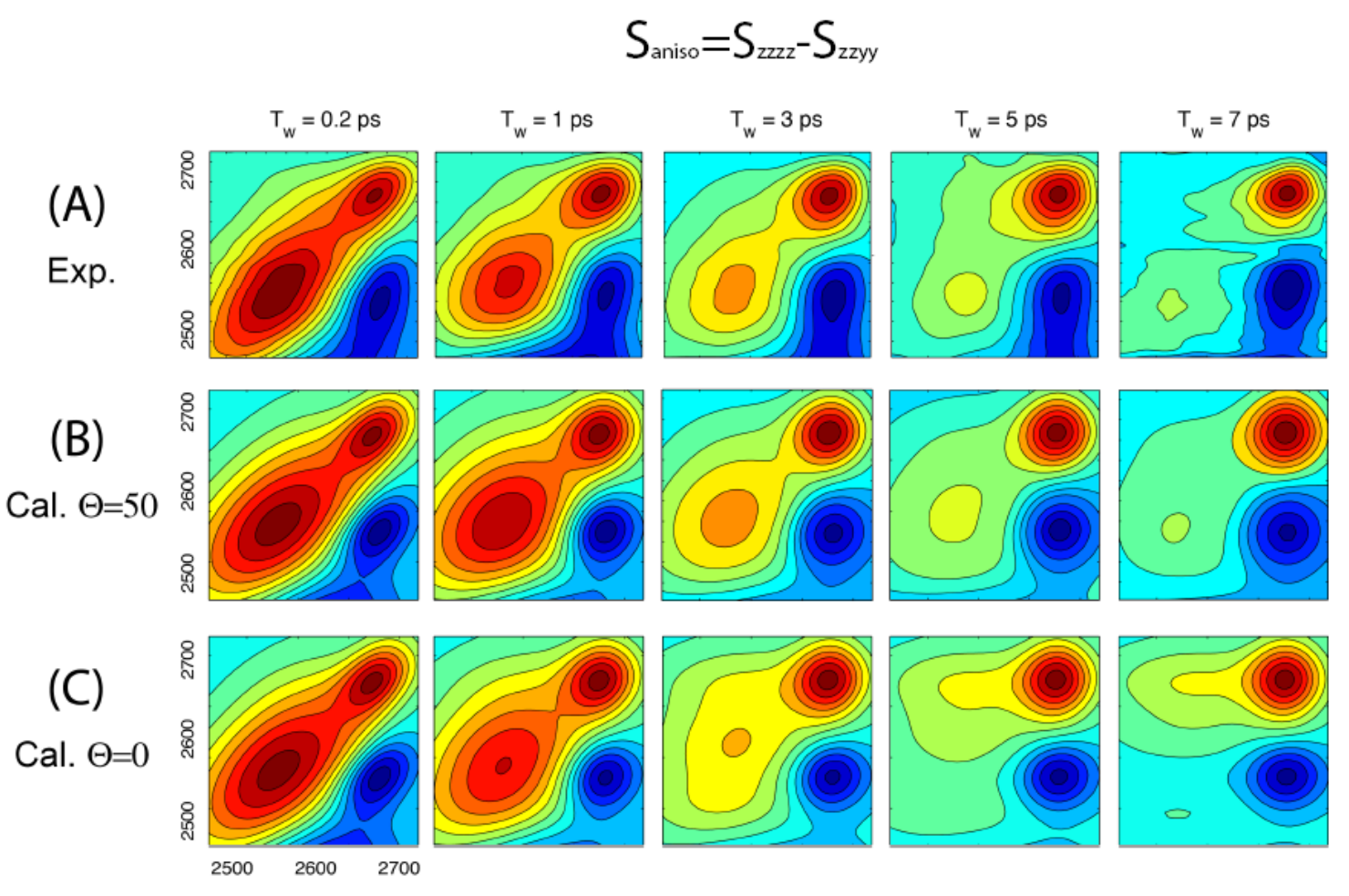

Fig. S5 The experimental anisotropic 2D spectra $S_{\text {aniso }}=S_{z z z z}-S_{z z y y}$ are shown in the first row (A), the calculated spectra using the response function and jump exchange kinetic model fitting results are shown in the second row (B), and the calculated spectra assuming $\Theta=0^{\circ}$ are shown in the third row $(\mathrm{C})$. 


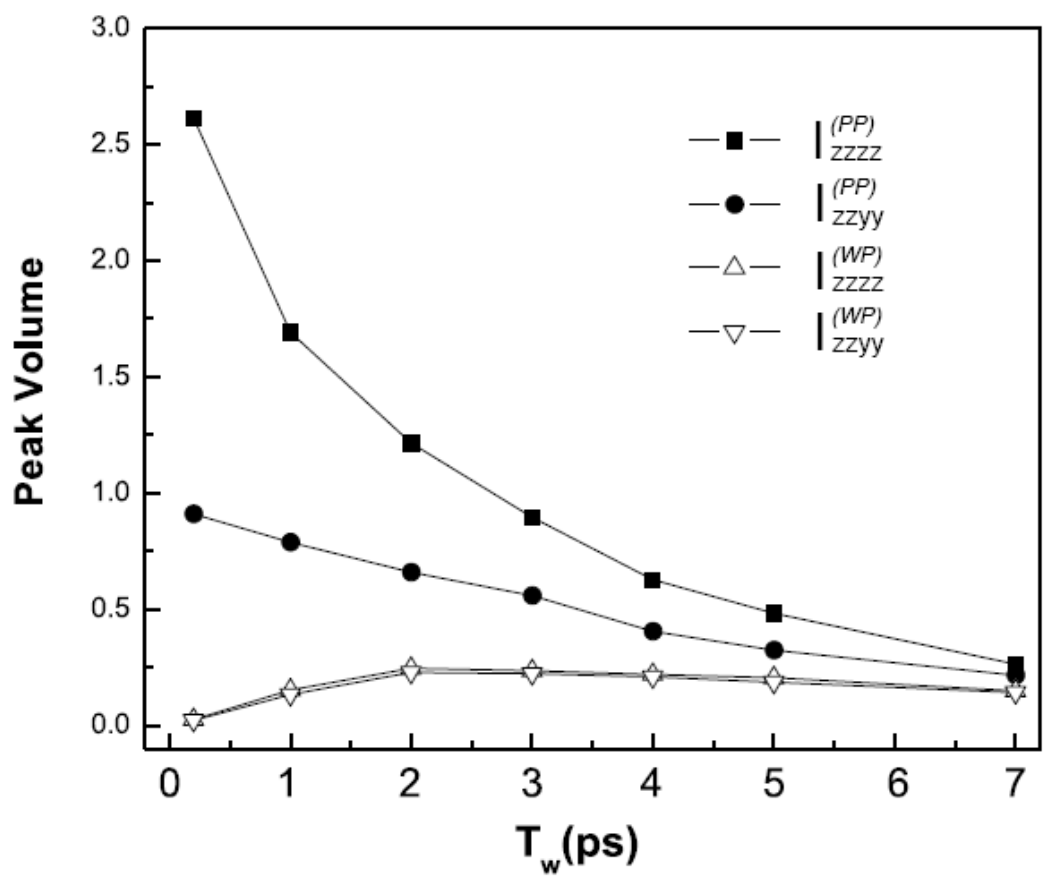

Fig. S6 Extracted peak volumes for the diagonal peak $I_{l l m m}^{(P P)}$, and cross-peak $I_{l l m m}^{(W P)}$. It is clearly seen that the diagonal peak and cross-peak volumes have significantly different polarization dependence. While the diagonal peak intensity has a large polarization dependence, the cross-peak intensity shows little polarization dependence. 

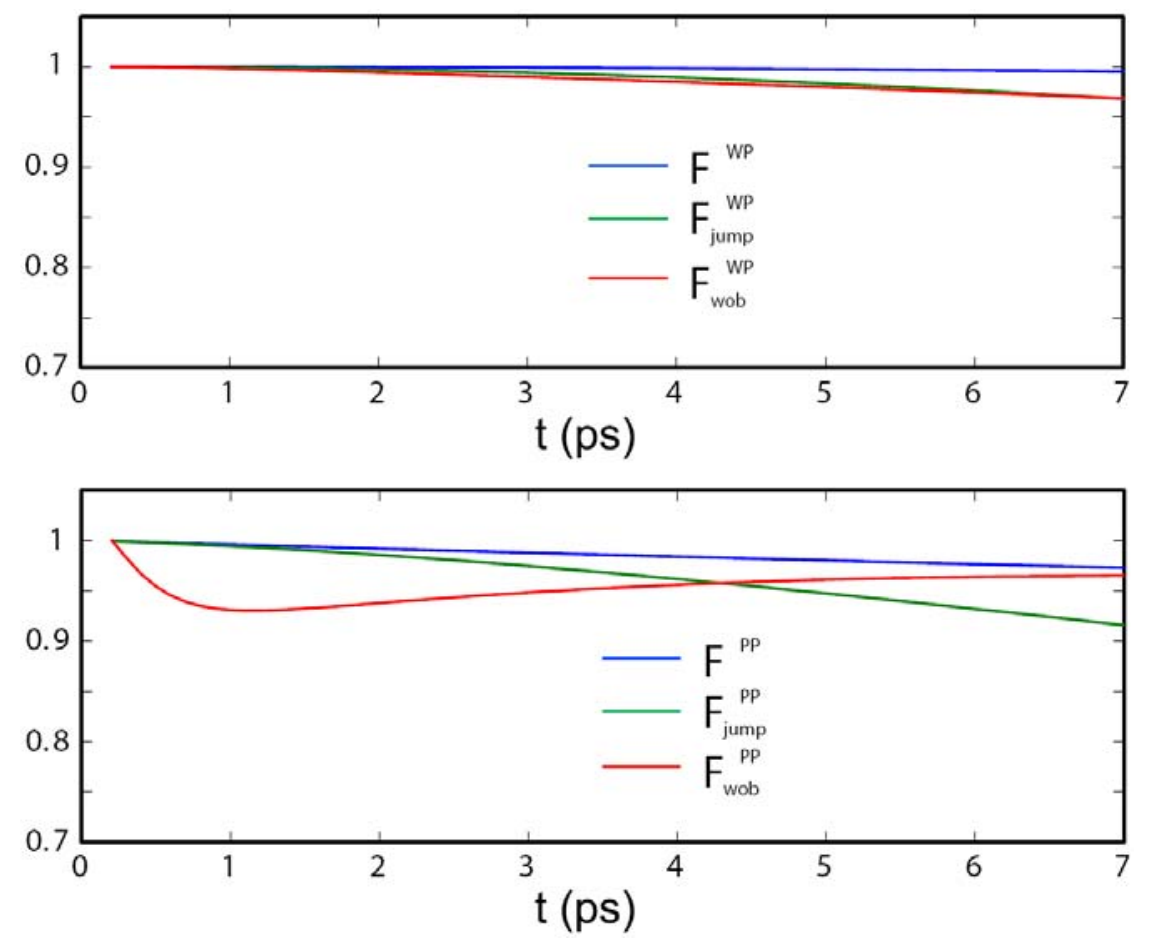

Fig. S7 $F^{i j}(t)$ functions calculated using the above three models (eqs. (12)-(17)), using the parameters from the best fit results. Note that these functions always reside within $10 \%$ of unity, validating their exclusion from the qualitative discussion. 
(A)

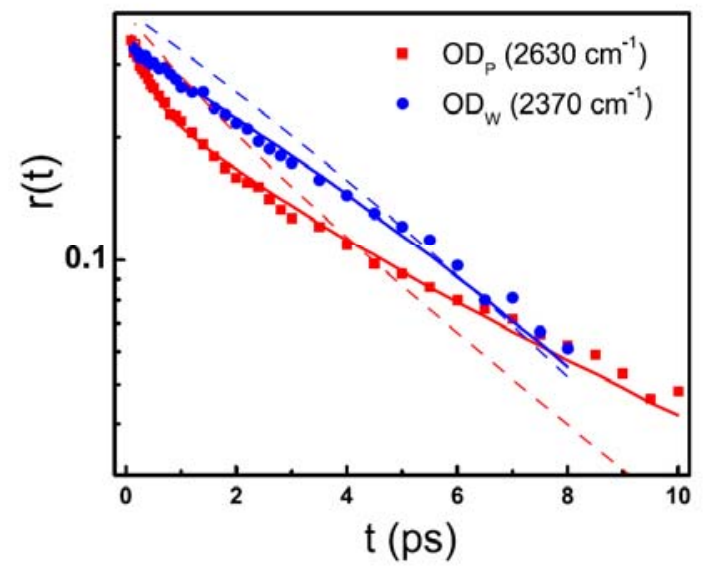

(B)

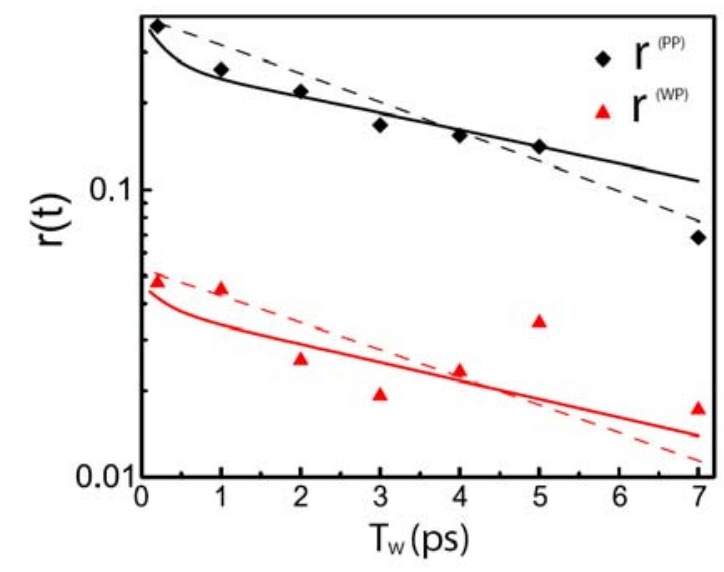

Fig. S8 Anisotropy data analysis. (A) Polarization resolved pump-probe measurements of the orientational dynamics of the $\mathrm{OD}_{\mathrm{W}}$ and $\mathrm{OD}_{\mathrm{P}}$ final state ensembles (dots). Solid lines are the best fit of the wobbling in a cone corrected JEK model (equations (8)-(11)). Dashed lines represent the JEK model with constant diffusion rates, which mismatch the data significantly. (B) 2DIR anisotropy data generated from the peak volumes of the diagonal and cross peaks (symbols). Solid lines are calculated with the wobbling in a cone corrected JEK model. Dashed lines are calculated with the JEK model.

Notice that although the wobbling model provides a more accurate representation of the experimental data, the correction to the extracted jump angle is small $\left(<2^{\circ}\right)$. This results for two reasons: (1) Wobbling reduces anisotropy globally for all the peaks (equation (16) and (17)), while the angular jump preferentially affects the cross-peak anisotropy (equation (14) and (15)). (2) For the large jump angles needed to fit the crosspeak anisotropy, $P_{2}(\cos \Theta)$ is very sensitive to $\Theta$. Thus moderate errors in the experimental data and uncertainties in the modeling lead to comparatively small uncertainties in $\Theta$. The wobbling in a cone corrected model modifies the cross-peak anisotropy to $\left\langle P_{2}(\cos \Theta)\right\rangle / \sqrt{S_{W}^{2} S_{P}^{2}}$. For the best fit shown by the solid lines in Fig. S8, $S_{W}^{2}=0.83$ and $S_{P}^{2}=0.65$, which only changes the value of $\langle\Theta\rangle$ from $50^{\circ}$ to $48.2^{\circ}$. Again this assumes a relatively symmetric distribution in $\Theta$, consistent with the CPMD simulation results shown in Fig. S9. Additonally, the cross-peak anisotropy is significantly smaller than the diagonal peak anisotropy, even for the earliest time delays. This strongly supports the conclusion that the angular jump occurs promptly, as seen in the CPMD simulation, and assumed in the model calculation. 

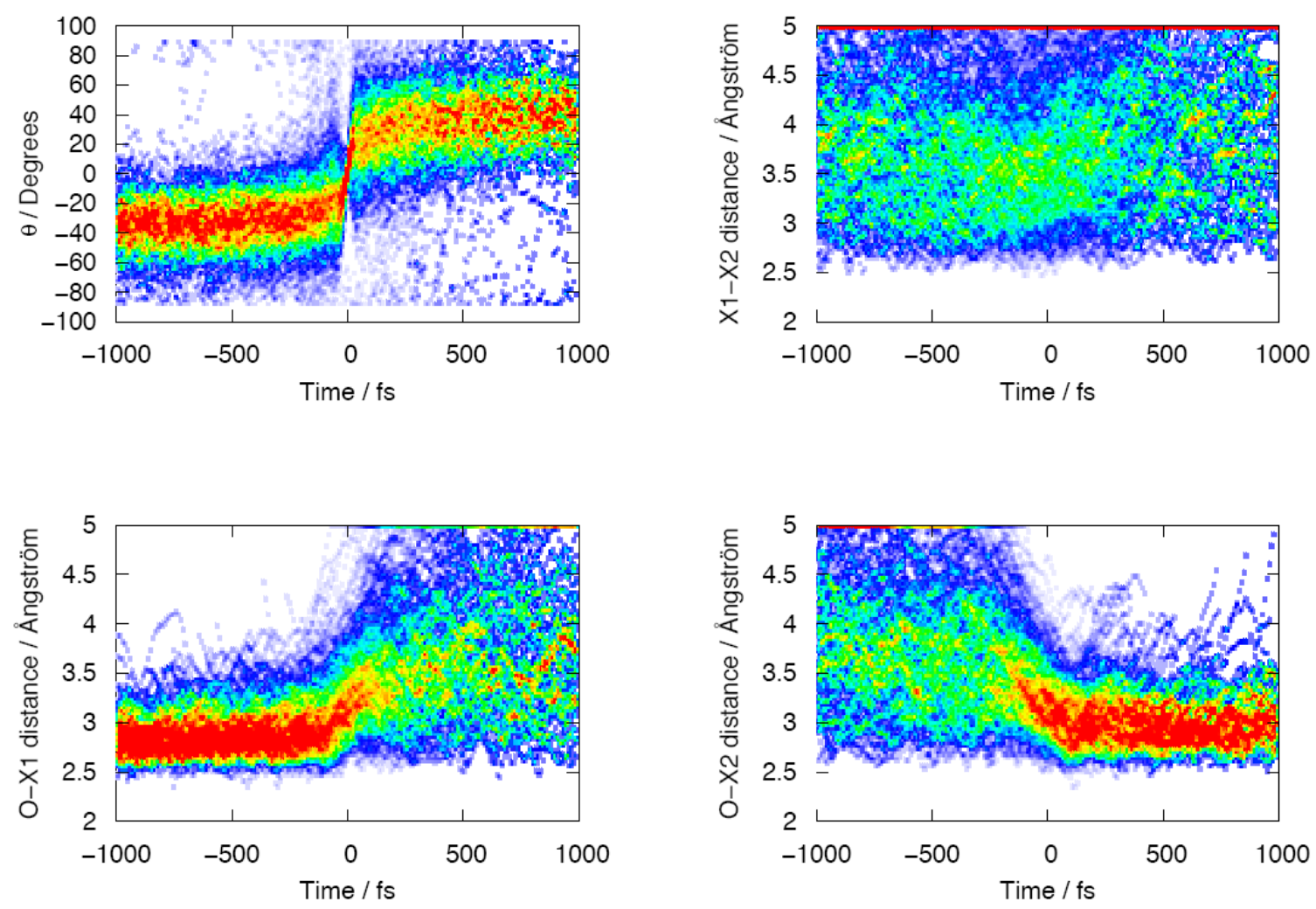

Fig. S9 Time dependent distributions of key structural degrees of freedom before and after an $\mathrm{OD}_{\mathrm{P}}$-to- $\mathrm{OD}_{\mathrm{W}}$ hydrogen bond exchange event where time zero corresponds to the mid-point of the exchange. The upper left plot shows the well defined jump angle, $\theta$, distribution before and after H-bond exchange. The upper right plot shows that the distance between the initial and final $\mathrm{H}$-bond accepting $\mathrm{O}$ atoms (X1-X2) does not have well defined distribution. As expected, the lower left plot shows that a well defined Hbond length between the $\mathrm{O}$ on the donating hydroxyl group and the $\mathrm{O}$ atom on the initial acceptor (O-X1) prior to, but not after, H-bond exchange. Also as expected, the lower right plot shows that a well defined $\mathrm{H}$-bond length between the $\mathrm{O}$ on the donating hydroxyl group and the $\mathrm{O}$ atom on the final acceptor $(\mathrm{O}-\mathrm{X} 2)$ after, but not prior to, $\mathrm{H}$ bond exchange. 

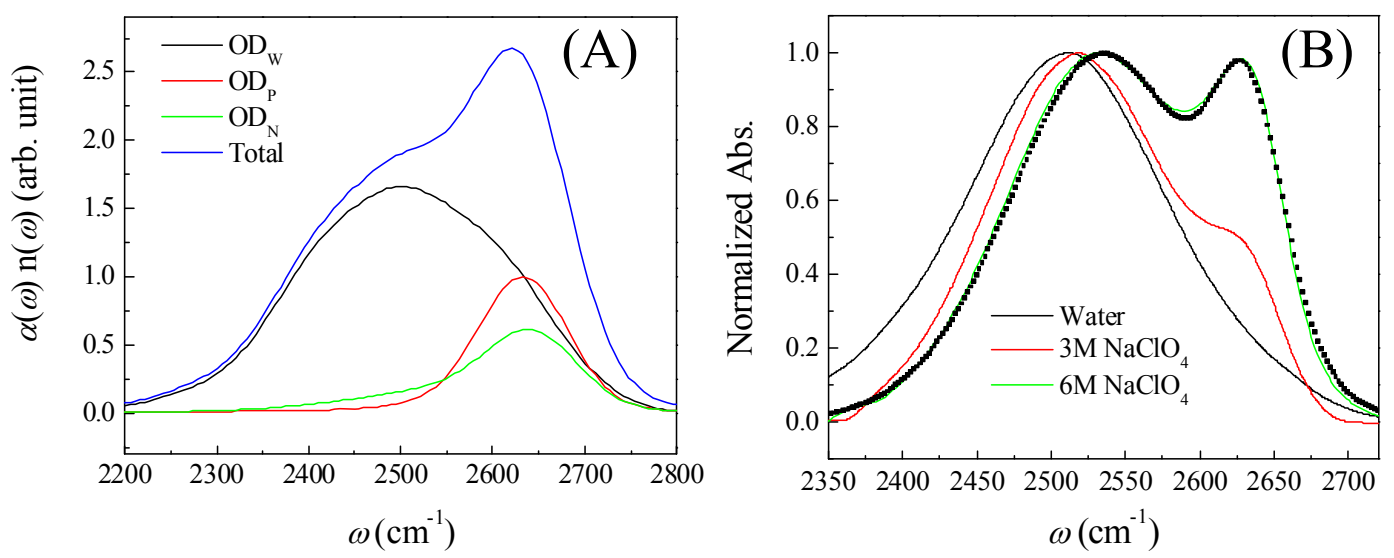

Fig. S10 (A) Linear IR spectra of the OD stretch of HOD in different H-bond configurations extracted from the CPMD simulation of aqueous $6 \mathrm{M} \mathrm{NaClO}_{4}$ solution. The low-frequency broad peak results from the OD groups that donate a H-bonded to other water molecules $\left(\mathrm{OD}_{\mathrm{W}}\right)$. The high frequency narrow peak results from the non-Hbonded OD groups and the OD groups that donate a H-bond to a perchlorate anion $\left(\mathrm{OD}_{\mathrm{P}}\right)$. The qualitative agreement between the FTIR spectrum of the OD stretch of HOD in aqueous $6 \mathrm{M} \mathrm{NaClO}_{4}$ solution (B) and the CPMD simulation results strongly support the spectroscopic assignments used in our analysis of the experimental data. (B) FTIR spectra of the OD stretch of HOD in aqueous $3 \mathrm{M}$ and $6 \mathrm{M} \mathrm{NaClO}_{4}$ solutions. The $\mathrm{H}_{2} \mathrm{O}$ background has been subtracted. FTIR spectrum of the OD stretch of HOD in isotopically mixed water is shown for comparison. 


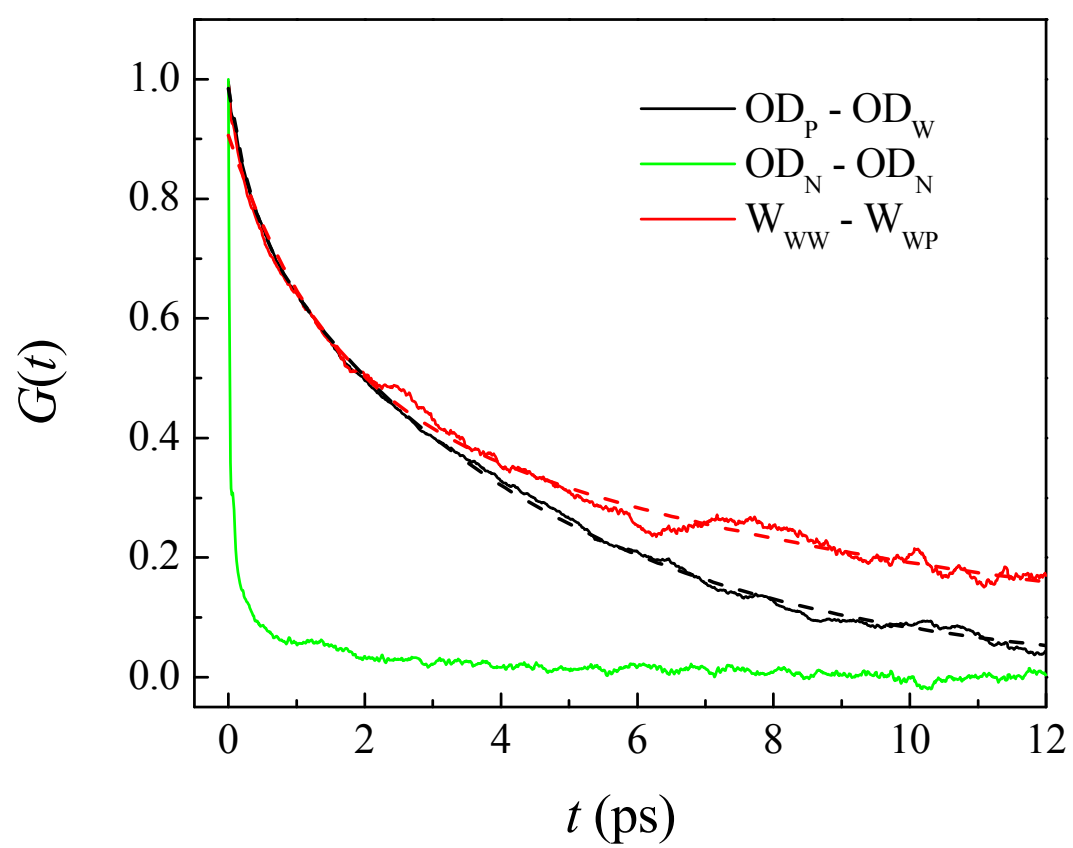

Fig. S11 Plot of three TCFs. The $\mathrm{OD}_{\mathrm{N}}-\mathrm{OD}_{\mathrm{N}} \mathrm{TACF}$ shown in green clearly demonstrates that non-H-bonded hydroxyl groups in $6 \mathrm{M} \mathrm{NaClO}_{4}$ solution decay extremely fast. This concurs with the short-lived non-H-bonded species observed in MD simulations of isotopically mixed water $(S 31)$. Consequently, the inclusion or exclusion of the non-Hbonded species has no impact on the slower relaxation dynamics observed in the simulations. We also plot the $\mathrm{OD}_{\mathrm{P}}-\mathrm{OD}_{\mathrm{W}} \mathrm{TCCF}$ in black. This correlation function has a slow decay that has been fit to a $4.4 \pm 0.5$ ps time constant, similar to the $6 \pm 1$ ps extracted from the experiment. The $\mathrm{W}_{\mathrm{WW}}-\mathrm{W}_{\mathrm{WP}} \mathrm{TCCF}$ in red represents a molecular correlation function, which we do not directly observe experimentally. 


\begin{tabular}{|l|l|l|l|l|l|l|l|}
\hline & & $\tau_{o r}(p s)$ & $S^{2}$ & $\tau_{\text {eff }}(p s)$ & $T_{1}(p s)$ & $\Theta($ degree $)$ & $\tau_{e x}(p s)$ \\
\hline \multirow{2}{*}{ JEK } & $O D_{P}$ & 4.6 & 1 & N/A & 5.24 & $50 \pm 4$ & $5 \pm 1$ \\
\cline { 2 - 6 } & $O D_{W}$ & 5.0 & 1 & N/A & 2.54 & & \\
\hline \multirow{2}{*}{$\begin{array}{l}\text { Wob- } \\
\text { JEK }\end{array}$} & $O D_{P}$ & 8.3 & 0.65 & 0.3 & 5.24 & $49 \pm 4$ & $6 \pm 1$ \\
\cline { 2 - 5 } & $O D_{W}$ & 6.0 & 0.83 & 0.03 & 2.54 & & \\
\hline
\end{tabular}

Table S1. Parameters extracted from best fitting by JEK and wobbling-corrected JEK model. The parameters are related to the modeling as: $\tau_{o r}=(6 D)^{-1}, T_{1}=k^{-1}$, and $\tau_{e x}=\left(k_{P-W}+k_{W-P}\right)^{-1}$. 


\section{References}

S1. M. Khalil, N. Demirdoven, A. Tokmakoff, J. Phys. Chem. A 107, 5258 (2003).

S2. $\quad$ S. Park, K. Kwak, M. D. Fayer, Laser Phys. Lett. 4, 704 (2007).

S3. $\quad$ Y. L. A. Rezus, H. J. Bakker, J. Chem. Phys. 123, 114502 (2005).

S4. D. Laage, J. T. Hynes, Science 311, 832 (2006).

S5. D. E. Moilanen et al., Proc. Natl. Acad. Sci. U.S.A 105, 5295 (April 8, 2008, 2008).

S6. $\quad$ S. Park, D. E. Moilanen, M. D. Fayer, J. Phys. Chem. B 112, 5279 (2008).

S7. $\quad$ G. Lipari, A. Szabo, Biophys. J. 30, 489 (1980).

S8. $\quad$ K. Kinosita, S. Kawato, A. Ikegami, Biophys. J. 20, 289 (1977).

S9. $\quad$ S. Park, M. Odelius, K. J. Gaffney, J. Phys. Chem. B 113, 7825 (2009).

S10. R. Car, M. Parrinello, Phys. Rev. Lett. 55, 2471 (1985).

S11. G. Heinje, W. A. P. Luck, K. Heinzinger, J. Phys. Chem. A. 91, 331 (1987).

S12. H. Krienke, D. Opalka, J. Phys. Chem. C 111, 15935 (2007).

S13. A. D. Becke, Phys. Rev. A 38, 3098 (1988).

S14. C. Lee, W. Yang, R. G. Parr, Phys. Rev. B. 37, 785 (1988).

S15. K. Toukan, A. Rahman, Phys. Rev. B. 31, 2643 (1985).

S16. N. Troullier, J. L. Martins, Phys. Rev. B. 43, 1993 (1991).

S17. L. Kleinman, D. M. Bylander, Phys. Rev. Lett. 48, 1425 (1982).

S18. S. Goedecker, M. Teter, J. Hutter, Phys. Rev. B. 54, 1703 (1996).

S19. C. Hartwigsen, S. Goedecker, J. Hutter, Phys. Rev. B. 58, 3641 (1998).

S20. S. Nos'e, J. Chem. Phys. 81, 511 (1984).

S21. S. Nos'e, Mol. Phys. 52, 255 (1984).

S22. W. G. Hoover, Phys. Rev. A. 31, 1695 (1985).

S23. I.-F. W. Kuo et al., J. Phys. Chem. B 108, 12990 (2004).

S24. B. Hetenyi, F. D. Angelis, P. Giannozzi, R. Car, J. Chem. Phys 120, 8632 (2004).

S25. R. G. Gordon, J. Chem. Phys. 43, 1307 (1965).

S26. D. W. Oxtoby, Annu. Rev. Phys. Chem. 32, 77 (1981).

S27. P. L. Silvestrelli, M. Bernasconi, M. Parrinello, Chem. Phys. Lett. 277, 478 (1997).

S28. K. N. Woods, H. Wiedemann, Chem. Phys. Lett. 393, 159 (2004).

S29. D. Laage, J. T. Hynes, Proc. Nat. Acad. Sci. 104, 11167 (2007).

S30. D. Chandler, Introduction to Modern Statistical Mechanics. (Oxford University Press, Inc, New York, 1987).

S31. J. D. Eaves et al., Proc. Natl. Acad. Sci. 102, 13019 (2005). 